\title{
Distorted Coulomb field of the scattered electron
}

\author{
H. D. Thomsen, ${ }^{1}$ J. Esberg, ${ }^{1}$ K. K. Andersen, ${ }^{1}$ M. D. Lund, ${ }^{1}$ H. Knudsen, ${ }^{1}$ U. I. Uggerh $ø j,{ }^{1}$ P. Sona, ${ }^{2}$ A. Mangiarotti, ${ }^{3}$ \\ T. J. Ketel, ${ }^{4}$ A. Dizdar, ${ }^{5}$ S. Ballestrero, ${ }^{6}$ and S. H. Connell ${ }^{6}$
}

(CERN NA63)

\author{
${ }^{1}$ Department of Physics and Astronomy, University of Aarhus, Denmark \\ ${ }^{2}$ University of Florence, Florence, Italy \\ ${ }^{3}$ Universidade de Coimbra, Portugal \\ ${ }^{4}$ Free University, Amsterdam, The Netherlands \\ ${ }^{5}$ University of Istanbul, Istanbul, Turkey \\ ${ }^{6}$ University of Johannesburg, Johannesburg, South Africa
}

(Received 4 August 2009; revised manuscript received 5 January 2010; published 10 March 2010)

\begin{abstract}
Experimental results for the radiation emission from ultrarelativistic electrons in targets of $0.03 \%-5 \%$ radiation length is presented. For the thinnest targets, the radiation emission is in accordance with the Bethe-Heitler formulation of bremsstrahlung, the target acting as a single scatterer. In this regime, the radiation intensity is proportional to the thickness. As the thickness increases, the distorted Coulomb field of the electron that is the result of the first scattering events, leads to a suppressed radiation emission per interaction, upon subsequent scattering events. In that case, the radiation intensity becomes proportional to a logarithmic function of the thickness, due to the suppression. Eventually, once the target becomes sufficiently thick, the entire radiation process becomes influenced by multiple scattering and the radiation intensity is again proportional to the thickness, but with a different constant of proportionality. The observed logarithmic thickness dependence of radiation intensity at intermediate values of the thickness can be directly interpreted as a manifestation of the distortion of the electron Coulomb field resulting from a scattering event. The Landau-Pomeranchuk-Migdal effect is explored with high primary energy using materials with low nuclear charge $(Z)$. Also, targets that should give rise to the claimed interference effect in high-energy radiation emission from a structured target of thin foils are investigated.
\end{abstract}

DOI: 10.1103/PhysRevD.81.052003

PACS numbers: $41.60 .-\mathrm{m}, 29.40 . \mathrm{Vj}$

\section{INTRODUCTION}

In the CERN NA63 experiment, we have investigated the emission of bremsstrahlung from ultrarelativistic electrons in targets of $0.03-5 \%$ radiation length. Within this range of thicknesses, for photon energies $0.2-3 \mathrm{GeV}$, and with the energy of the impinging electron of $149 \mathrm{GeV}$, the dependence of radiation intensity on thickness is shown to be a simple logarithmic dependence in the scattering region between the Bethe-Heitler $(\mathrm{BH})$ and LandauPomeranchuk-Migdal (LPM) regimes. We have also explored the LPM regime using very light materials, i.e. with low nuclear charge $(Z)$, and searched for the claimed interference effect in high-energy radiation emission from a structured target of thin foils [1-5]—a so-called structured or sandwich target, both at a primary energy of $207 \mathrm{GeV}$. The latter effect is due to the formation length of the $\mathrm{GeV}$ photons extending across the $\simeq 90$ micron "gap" between the thin Ta foils and has no direct relation to transition radiation.

\section{Visualization of radiation by means of electric field lines}

In Fig. 1, we show a picture of the distorted Coulomb field of the electron immediately following one and two scattering events, generated on the basis of the procedure outlined in [6] for the visualization of radiation by means of electric field lines. Such a fieldline interpretation is also applicable for the magnetic field [7], producing a fully classical picture of radiation from the tangential components. Two things are clear from this picture: After the first scattering event, the Coulomb field of the electron is distorted due to the finite propagation speed of disturbances of the electric field. Also, due to this distortion, a subsequent scattering event may lead to closely spaced tangential component field lines pointing in opposite directions, cf. Figure 1(b). From the similarity theorem of Fourier decomposition, the low frequency component of the radiation passing the observer-the one with the longest separation in time-will therefore contain field vectors that give mutual cancellation. Thus the radiation intensity per unit length for two scattering events in close succession is suppressed at low frequencies. Therefore, the fieldline picture not only gives information on the direction and intensity but also on the spectral composition, albeit in a qualitative manner only.

The distortion of the Coulomb field is essentially the basis of the theory for suppressed bremsstrahlung emission from a finite target, developed by Shul'ga and Fomin (SF) 


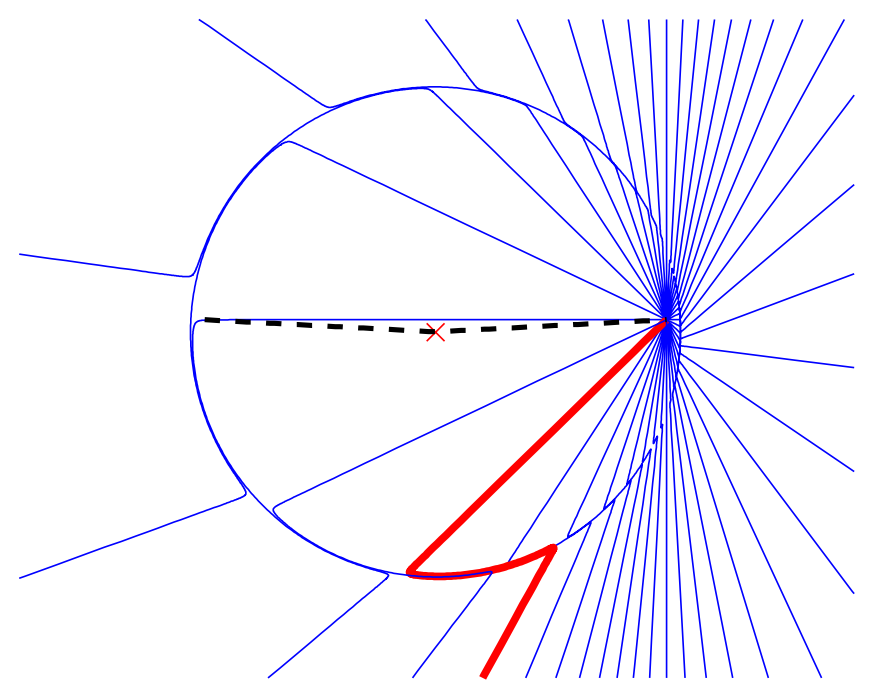

(a) Single Scattering.

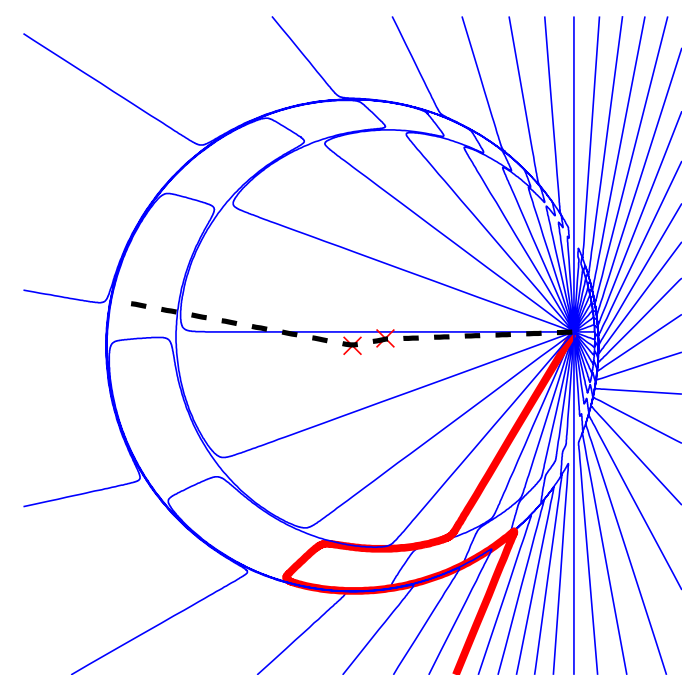

(b) Double Scattering.

FIG. 1 (color online). The electric field lines shortly after the electron has scattered once (a) and twice (b), respectively. For illustration purposes, the velocity of the particle has been set to $\beta=0.9$. The tangential component of the field lines can be interpreted as the radiation field, propagating outwards at the speed of light [6,7]. As seen from one of the field lines (indicated by the thick red line), the rapid succession of scattering events may lead to closely spaced tangential component field lines pointing in opposite directions. To the observer, these field lines cancel each other for low frequencies.

[8,9], based on the concept of the semibare electron introduced by Feinberg [10]. The latter concept, although appealing, is questionable [11], but alternative routes to bremsstrahlung emission from a finite target by Blankenbecler and Drell (BD) [1] and Baier and Katkov (BK) [12] show the same behavior. We present in the following an experiment that shows exactly this predicted suppression, going from the linear behavior at ultrathin targets through a logarithmic dependence resulting from the suppression, to a weaker linear rise with thickness.

\section{THEORY}

\section{A. Bethe-Heitler}

The cross section for emission of radiation of energy $\hbar \omega$ in an amorphous foil-incoherent bremsstrahlung — can be found from the $\mathrm{BH}$ formula [13]. The $\mathrm{BH}$ cross section is derived for scattering off a single atom, but applies to extended targets as long as coherence effects can be neglected. In this case, the radiation power spectrum (or the logarithmically binned photon spectrum) $\hbar \omega \times$ $d N_{\gamma} / d \hbar \omega=d N_{\gamma} / d \ln \hbar \omega$ from a number of electrons $N_{e}$ with energy $E$ traversing a foil of longitudinal thickness $\Delta t$ is in the full screening limit $\left(E \gg m c^{2}\right.$, where $m$ is the electron mass and $c$ the speed of light) approximately given by

$$
\begin{aligned}
\frac{\hbar \omega}{N_{e}} \frac{d N_{\gamma}}{d \hbar \omega} \simeq & \frac{16 n \Delta t Z(Z+1) \alpha r_{e}^{2}}{3}\left[1-y+\frac{3}{4} y^{2}\right] \\
& \times \ln \left(183 Z^{-1 / 3}\right) \\
= & \frac{4}{3} \frac{\Delta t}{X_{0}}\left[1-y+\frac{3}{4} y^{2}\right],
\end{aligned}
$$

where $Z$ is the material's nuclear charge, $n$ the number density of the scattering atoms, $\alpha$ the fine structure constant, $y=\hbar \omega / E, X_{0}$ the radiation length, and $r_{e}=\alpha \hbar / m c$ the classical electron radius. By writing $Z(Z+1)=Z^{2}+$ $Z$ (and not $Z^{2}$ ), the contribution from the atomic electrons has been included [14]. The radiated intensity is directly proportional to the target thickness, i.e. the intensity per encounter is constant.

\section{B. The Landau-Pomeranchuk-Migdal effect}

Because of the uncertainty in the longitudinal recoil momentum taken by the nucleus from which a high energy electron scatters during bremsstrahlung emission, there is a corresponding distance over which the photon can be considered "formed," the so-called formation length $\ell_{\mathrm{f}}$. It is approximately equal to the distance of travel necessary for the electron to "lag behind" the photon by one reduced wavelength, i.e. loosely speaking, the photon has "separated" from the emitting particle [15,16] (see also [17]). Even for $\mathrm{GeV}$ photons the formation length can be of macroscopic dimensions if the energy of the emitting particle is large enough

$$
\ell_{\mathrm{f}}=\frac{2 E(E-\hbar \omega)}{m^{2} c^{3} \omega}=\frac{2 \gamma^{2} c}{\omega^{*}} \quad \omega^{*}=\omega \frac{E}{E-\hbar \omega},
$$

where $\gamma$ is the Lorentz factor, $\gamma=E / m c^{2}$. The formation length is approximately equal to $2 \gamma^{2} c / \omega$ for soft photons, $\hbar \omega \ll E$. The finite formation length of the photon-the length over which the lepton may interact again resulting in enhanced or suppressed radiation emission-is the basis of the LPM effect. In short, the LPM effect appears for photon 
energies where the multiple scattering over the formation length $\theta_{\mathrm{f}}$ deflects the radiating particle outside the radiation cone with opening angle of the order of the inverse of the Lorentz factor, $1 / \gamma$. The length over which an ensemble of particles acquires an RMS scattering angle of $\theta_{\mathrm{f}}=1 / \gamma$ in an amorphous material due to multiple Coulomb scattering is given by the multiple scattering length $\ell_{\gamma}=\alpha X_{0} / 4 \pi$. This leads to a "threshold" of the LPM effect appearing at energies $\hbar \omega \lesssim \hbar \omega_{\mathrm{LPM}}=$ $E^{2} /\left(E+E_{\mathrm{LPM}}\right) \quad$ where $\quad E_{\mathrm{LPM}}=m c^{2} X_{0} / 4 \pi a_{0}=$ $7.684 \cdot X_{0} \mathrm{TeV} / \mathrm{cm}$ and $a_{0}$ is the Bohr radius. In the $\mathrm{BH}$ case, the power spectrum is nearly constant with photon energy, whereas for the LPM effect it is proportional to $\sqrt{\hbar \omega}$. Like in the $\mathrm{BH}$ regime, the power spectrum is directly proportional to target thickness, i.e. the intensity per encounter is constant, although at a reduced level compared to BH. Details about recent experiments on the LPM effect can be found in [18-20].

The formation length is fundamental to a number of phenomena in radiation physics, ranging from strong enhancements in crystals [17] to suppression effects in dense matter [18]. Furthermore, insights concerning formation lengths are applied in many other branches of physics, e.g. in the strong interaction [21], plasma-wave acceleration [22], or radiation from the electrosphere of hypothetical strange stars $[23,24]$. As the electrosphere of strange stars is $\simeq 10^{-12} \mathrm{~m}$ thick, thin target corrections to the standard LPM theory hitherto applied may be relevant.

Both the "traditional" Migdal approach [25] and the modern treatment by Baier and Katkov display inaccuracies, i.e. a possible lack of applicability in low $Z$ targets. Moreover, the LPM effect has been shown to have a significant impact on giant air showers for energies in the $\mathrm{EeV}$ range-evidently processes in a low $Z$ material.

In his review paper on the LPM effect from 1999 [18], Spencer Klein stated among the explanations for a small, but significant discrepancy found for carbon with electrons at $25 \mathrm{GeV}$ that "it is also possible that Migdal's theory may be inadequate for lighter targets." Likewise, in the CERN experiments [20], where carbon was used as a calibration target, the systematic deviations from the expected values for $E_{\mathrm{LPM}}$ could possibly be explained by an insufficient theoretical description of carbon.

\section{Longitudinal density effect}

The photon can also cause loss of coherence. In a medium with electronic plasma frequency $\omega_{p}=$ $\sqrt{4 \pi N Z e^{2} / m}$ and index of refraction $n=1-\omega_{p}^{2} / 2 \omega^{2}$ $\left(\omega \gg \omega_{p}\right)$, the photon travels with reduced speed $c / n$. This modifies the vacuum formation length from $\ell_{\mathrm{f}}$ to $\ell_{\mathrm{f} \epsilon}$ [18]

$$
\frac{1}{\ell_{\mathrm{f} \epsilon}} \simeq \frac{\omega}{2 \gamma^{2} c}+\frac{\omega_{p}^{2}}{2 \omega c} \Leftrightarrow \ell_{\mathrm{f} \epsilon} \simeq \frac{\ell_{\mathrm{f}} \ell_{\mathrm{df}}}{\ell_{\mathrm{f}}+\ell_{\mathrm{df}}}
$$

where $\ell_{\mathrm{df}}=2 \omega c / \omega_{p}^{2}$. Clearly, the formation lengths larger than $\ell_{\mathrm{df}}$ are effectively cut away-much in analogy to the density effect concerning ionization energy loss [26], where large transverse distances are effectively cut away. This longitudinal density effect is attributed to TerMikaelian [16] and causes a suppression at $\hbar \omega<\hbar \omega_{\text {LD }}=$ $\gamma \hbar \omega_{p}$. Here $d \sigma_{\mathrm{LD}} / d \ln \hbar \omega \propto(\hbar \omega)^{2}$, much different from the other suppression mechanisms.

\section{The Ternovskii-Shul'ga-Fomin effect}

The LPM theory applies only to a semi-infinite target. For particle energies of a few hundred $\mathrm{GeV}$, the formation length of a few hundred $\mathrm{MeV}$ photon becomes more than $100 \mu \mathrm{m}$ long. In this case, e.g. a $30 \mu \mathrm{m}$ thick target can clearly not be considered semi-infinite. By equating $\Delta t$ and $\ell_{\mathrm{f}}$, a threshold at which the LPM theory will become insufficient can be estimated

$$
\hbar \omega<\hbar \omega_{\mathrm{TSF}}=\frac{E}{1+\frac{\Delta t}{2 \gamma \hbar_{c}}},
$$

where $\lambda_{c}=\hbar / m c$ is the reduced Compton wavelength. This regime was first considered by Ternovskii [27] and later a classical theory for thin targets was developed by Shul'ga and Fomin [8,9] giving name to the TernovskiiShul'ga-Fomin (TSF) effect. Here, the differential cross section has the photon energy dependence of $\mathrm{BH}$ again, but is reduced in magnitude by approximately [28]

$$
\kappa \simeq \frac{\Delta t}{6 \ell_{\gamma}\left[\ln \left(\Delta t / \ell_{\gamma}\right)-1\right]} .
$$

In a later paper [29], SF included the corrections due to quantum recoil. Meanwhile, Baier and Katkov developed a full quantum theory of the LPM effect with Coulomb corrections, including emission in a thin foil [12]. Moreover, Blankenbecler and Drell [1] supplemented by a calculation based on their eikonal approximation to beamstrahlung phenomena. The aim of the latter theory was to describe the LPM bremsstrahlung spectrum and provide a smooth connection to the $\mathrm{BH}$ regime. These three approaches give almost identical results concerning radiation emission from a thin foil. The radiated intensity in the intermediate regime between BH and LPM becomes - for a fixed photon energy - a logarithmic function of the thickness.

The situation of thin targets was previously considered in connection with experimental investigations of the LPM effect at SLAC [30] where it turned out [31] that edge effects were important (see also [32]). These experiments, however, did not establish the thickness dependence directly, but showed good agreement as a function of photon energy, for one thickness, with the mentioned theories supporting logarithmic thickness dependence. The expected logarithmic tendency has later been further elaborated upon [12,33]. 
For practical reasons, which will be explained later, we consider in our experiment a target consisting of $N$ independent foils of thickness $\delta t$. The total thickness is given by $\Delta t=\sum \delta t=N\langle\delta t\rangle$. The simplest logarithmic thickness dependent power spectrum for a single foil of thickness $\delta t$ per electron with proper convergence to the $\mathrm{BH}$ limit as $\delta t \rightarrow 0$ would be

$$
\left.\frac{1}{N_{e}} \hbar \omega \frac{d N_{\gamma}}{d \hbar \omega}\right|_{1}=\left.\frac{1}{N_{e}} \frac{d N_{\gamma}}{d \ln \hbar \omega}\right|_{1}=\frac{a}{X_{0}} \times \frac{\ln (b \times \delta t+1)}{b},
$$

where the subscript indicates the number of independent foils being considered. The right-hand side (RHS) will converge towards $a \times \delta t / X_{0}$ as $\delta t \rightarrow 0$. By comparing with the left-hand side, the factor $a$ is thus seen to represent the unitless value of the $\mathrm{BH}$ power spectrum $d N_{\gamma} / d \ln \hbar \omega=\hbar \omega \times d N_{\gamma} / d \hbar \omega$ normalized to thickness $\left(\delta t / X_{0}\right)$ and number of particles $\left(N_{e}\right)$. This factor is of order unity and slightly dependent on $\hbar \omega$. The constant $b$ describes the rate at which the thickness normalized power spectrum level decreases with thickness-i.e. at $\delta t=1 / b$ the power spectrum is reduced by a factor $\ln (2)$ compared to the $\mathrm{BH}$ value $a$. Both sides of the equation above are unitless, while $b$ has dimension of inverse length. When considering an assembly of $N$ foils, the contributions from each foil add incoherently (if interference effects can be neglected) and the thickness is replaced with the mean thickness $\delta t \rightarrow\langle\delta t\rangle$

$$
\begin{aligned}
\left.\frac{1}{N_{e}} \frac{d N_{\gamma}}{d \ln \hbar \omega}\right|_{N} & =N \times\left.\frac{1}{N_{e}} \frac{d N_{\gamma}}{d \ln \hbar \omega}\right|_{1} \\
\left.\frac{X_{0}}{\Delta t} \frac{1}{N_{e}} \frac{d N_{\gamma}}{d \ln \hbar \omega}\right|_{N} & =N \times \frac{a}{\Delta t} \times \frac{\ln (b \times\langle\delta t\rangle+1)}{b} \\
& =a \times \frac{\ln (b \times\langle\delta t\rangle+1)}{b \times\langle\delta t\rangle},
\end{aligned}
$$

where $\Delta t=\sum \delta t=N\langle\delta t\rangle$ has been employed. The logarithmic expression in the Appendix is shown to be the major component of the theory for thin foils in [2], from which

$$
b_{\mathrm{B}}=2 \pi / 3 \alpha X_{0} \simeq 287 / X_{0},
$$

is found. The simple expression also closely approximates (accuracy $\leqslant 3 \%$ ) the much more complex theoretical expression [[33], Eq. (4.9)].

Like many of the other suppressions, this mechanism has been considered in its QCD analogue, "bremsstrahlung" gluons from a gluon traversing a finite nucleus [34] during a heavy ion collision.

\section{E. The sandwich effect}

A structured or "sandwich" target is composed of a sequence of $N$ equidistant foils of equal thicknesses, where typically the separation between the target segments is larger than their thickness. Put differently, it can be thought of as an initially homogeneous target, where sectionscorresponding to what eventually becomes the separation-have been removed. In Blankenbecler's theory [2,3] (see also [4]), interference mechanisms are considered for structured targets of up to 10 segments. It is shown that the photon spectrum is clearly developing a peak where the formation length is approximately equal to the distance between the centers of the plates [2]. Even though these calculations are performed only for $25 \mathrm{GeV}$ (and in a single case $50 \mathrm{GeV}$ ), we expect that this observation-see below-does apply to the general case. Furthermore, the interference over the distance between the backside of one plate and the frontside of the following plate must lead to the onset of the resonance peak. Therefore, Eq. (2) can be inverted setting the formation length $\ell_{\mathrm{f}}$ equal to the target spacing or gap width $\delta g$ leading to an onset of resonance at a photon energy

$$
\hbar \omega<\hbar \omega_{\mathrm{r}}=\frac{E}{1+\frac{\delta g}{2 \gamma \hbar_{c}}},
$$

which for $E \gg \hbar \omega_{\mathrm{r}}$ coincides with the "resonance condition" in a stratified medium [[16], Eq. (28.10)].

In [5], Baier and Katkov treat the radiation emission from a stack of thin foils, including the LPM, polarization effects and emission from the target boundaries (transition radiation). For the general case of $N$ foils, however, they only give an explicit formula for the strong scattering, large spacing case where, in their notation, $b \ll 1$ and $T=\left(l_{1}+\right.$ $\left.l_{2}\right) / \ell_{\mathrm{f}} \gg 1, l_{1}$ being the target segment thickness, $l_{2}$ the segment spacing [their Eq. (2.49)] and $b=\alpha X_{0} / 2 \pi l_{1}$ the scattering variable. In our case, $b=0.48$ does not fulfill the requirement, and the length variable $T \gg{ } /$ for photon energies in the interesting region, for instance $T \leq 0.94$ for $\hbar \omega \leq 1 \mathrm{GeV}$ with $l_{2}=90 \mu \mathrm{m}$ separations of $l_{1}=$ $10 \mu \mathrm{m}$ Ta targets at $207 \mathrm{GeV}$. The theory of Baier and Katkov is thus not directly applicable.

As stated in the introduction, from the fieldline picture it is found that the radiation intensity per unit length for scattering events in close succession is suppressed at low frequencies. Furthermore, the frequency interval which is suppressed, depends on the distance between the scattering centers-the longer this distance, the lower the suppressed frequency. It is therefore to be expected that the removal of possible scattering centers - as in the alternative view of the structured target-results in an "alleviation" of the suppression. The same picture is applicable for the TSF effect, where the suppression continues down to frequencies where the formation length extends out of the target, from which point the suppression "lifts" and the radiation level becomes a certain fraction of the unsuppressed (Bethe-Heitler) intensity. Thus, a comparison of a structured target-with an incomplete suppression-to a homogeneous one of the same thickness where the suppression is complete, will display an enhancement in radiation intensity at frequencies corresponding to the target spacings. 
However, such an enhancement is in a sense not really an enhancement, but a "lifted" suppression, and the radiation intensity thus never exceeds the unsuppressed $(\mathrm{BH})$ intensity. The latter observation is in accordance with our calculations based on the BD theory, performed for various energies and target constructions. Finally, in the view just presented, the structured target "resonance" effect can be seen as a suppression-alleviation-suppression effect (where larger distances covered by the particle into the structure converts into lower frequencies), using the TSF picture of the alleviated suppression.

The treatment in [1] does not include any correlation between phase and amplitude in the eikonal wave function. The later study by Blankenbecler [3] has shown that such a correlation reduces the soft-photon level of the spectrum by $5 \%-15 \%$ [18] and the shape is also slightly different. The interference structure between two foils prevails, although it is shifted slightly towards lower photon energies.

We present a search for the interference that theory predicts when the formation zone extends across a gap between two foils in our experiment.

\section{SETUP}

The experiment was performed in the $\mathrm{H} 4$ beam line of the CERN Super Proton Synchrotron (SPS) in a tertiary beam of electrons with variable energy in the range 10$300 \mathrm{GeV}$, but with low intensities at very low or very high energies. Beams of the energies 149 and $207 \mathrm{GeV}$ were used in the experiment. The energies were chosen as a trade off between having macroscopic formation lengths during emission of photons with energies of up to a few $\mathrm{GeV}$ and retaining acceptable beam intensities. A schematic drawing of the setup is shown in Fig. 2. The electron beam was defined by the scintillator counters $\mathrm{S} 1, \mathrm{~S} 2, \overline{\mathrm{S} 3}$, where the latter is a $\varnothing 9 \mathrm{~mm}$ hole scintillator (used in veto). The hereby accepted beam is centered on the target, and, after having traversed this, the electrons were swept away by a dipole magnet B16. This method of separation inevitably introduces Synchrotron Radiation (SR), the implication of which will be discussed in Sec. IVA. To be able to correct for the background, an empty target run was performed at both energies. Vacuum tubes and a He-bag (not shown in figure) were used where possible to reduce back- ground to a minimum in the $20 \mathrm{~m}$ long setup. The beam spot size at the calorimeter position was about $\varnothing 12 \mathrm{~mm}$, judging from previous measurements of the typical beam divergence using drift chambers [20]. The data was recorded using an event-based CAMAC system making offline event selection possible. The count rate was $\simeq 1.2 \times 10^{3}$ electron triggers $\mathrm{S} 1 \cdot \mathrm{S} 2 \cdot \overline{\mathrm{S} 3}$ per second during the $10 \mathrm{~s}$ long pulse repeated every $33 \mathrm{~s}$, for all targets.

\section{A. Targets}

All targets consisted of $N$ layers of disc-shaped foils of $\varnothing$ $25 \mathrm{~mm}$ and varying thickness $\delta t$ and material. The number of layers in a target was set such that the total target thickness $\Delta t$ would correspond as closely as possible to $100 \mu \mathrm{m}$ of tantalum $\left(2.44 \% X_{0}\right)$. The choice of total thickness was a trade off between obtaining an acceptable signal-to-background ratio and keeping multiphoton events at a minimum. The influence of the latter is already substantial $(\sim 20 \%)$ at this thickness [18,20], as will be discussed in Sec. VA 1. Keeping target thickness in units of $X_{0}$ almost constant would make the contribution from multiphoton events the same in all spectra, as the correction-essentially a shape function - should be a polynomial of the variable $\Delta t / X_{0}$. The thickness of the many foils was carefully determined. With the thinner ones, this was done by measuring their weight and transverse dimensions, whereas the thicker ones could be measured directly with acceptable accuracy using a micrometer gauge. In this way, $\delta t$ could be determined to $\simeq 1 \%$, cf. Table I. The aluminum, carbon, and tantalum foils are of $99.999 \%, 99.95 \%$, and $99.9 \%$ purity, respectively, according to the supplier.

Interference between adjacent foils was avoided (except for the sandwich measurements, cf. Sec. V C) by placing $1 \mathrm{~mm}$ precision steel ring spacers between neighboring foils. Each spacer has a $\varnothing 18 \mathrm{~mm}$ concentric hole, hence the spacers in the assembly did not contribute to the target material in the beam accepted by $\mathrm{S} 1 \cdot \mathrm{S} 1 \cdot \overline{\mathrm{S} 3}$. Having adjacent foils effectively separated by a gap $\delta g=1 \mathrm{~mm}$ of air, formation length related interference phenomena are excluded as long as $\ell_{\mathrm{f}} \lesssim \delta g$, corresponding to $\hbar \omega \geqslant$ $34 \mathrm{MeV}(65 \mathrm{MeV})$ using the $149 \mathrm{GeV}(207 \mathrm{GeV})$ beam, cf. Equation (9). The target assemblies are in the following labeled by the nomenclature $N \times \mathrm{A} \delta t[\mu \mathrm{m}] \mathrm{B} \delta g[\mu \mathrm{m}]$, where $A$ and $B$ refer to the foil and gap material, respec-

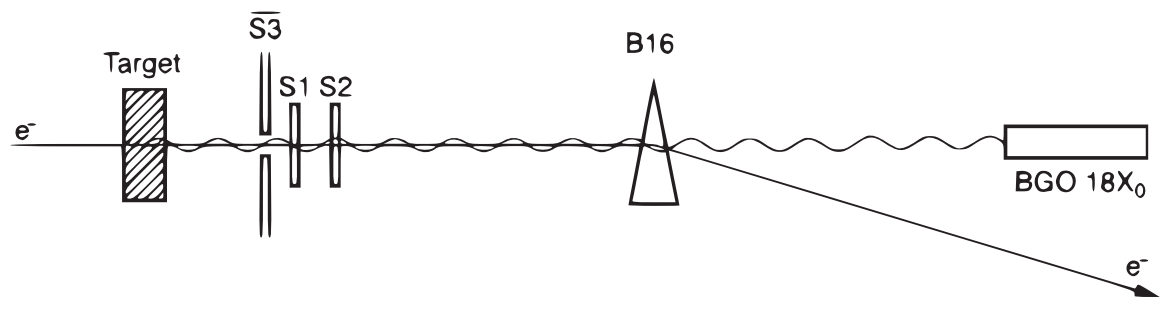

FIG. 2. A schematic drawing of the setup used in the experiment. The total length of the setup is about $20 \mathrm{~m}$. 
TABLE I. Nominal and measured foil thicknesses. The measured values are all within suppliers tolerances, which are listed as uncertainties on $\delta t_{\text {Nom. }}$.

\begin{tabular}{lcccc}
\hline \hline Element & $N$ & $\delta t_{\text {Nom }}[\mu \mathrm{m}]$ & $\langle\delta t\rangle_{\text {Meas }}[\mu \mathrm{m}]$ & $\Delta t_{\text {Meas }}[\mu \mathrm{m}]$ \\
\hline $\mathrm{C}$ & 1 & $5000 \pm 500$ & $4843 \pm 24$ & $4843 \pm 24$ \\
$\mathrm{Al}$ & 80 & $25.0 \pm 3.8$ & $25.56 \pm 0.03$ & $2045 \pm 3$ \\
& 1 & $2000 \pm 50$ & $2020 \pm 20$ & $2020 \pm 20$ \\
$\mathrm{Ta}$ & 20 & $5.0 \pm 1.3$ & $4.41 \pm 0.02$ & $88.2 \pm 0.5$ \\
& 17 & $6.0 \pm 1.5$ & $5.94 \pm 0.08$ & $101 \pm 1$ \\
& 14 & $7.5 \pm 1.9$ & $8.43 \pm 0.07$ & $118 \pm 1$ \\
& 11 & $9.0 \pm 2.3$ & $8.03 \pm 0.11$ & $88.4 \pm 1.2$ \\
& 10 & $10.0 \pm 1.5$ & $9.74 \pm 0.11$ & $97.4 \pm 1.1$ \\
& 8 & $12.5 \pm 1.9$ & $11.7 \pm 0.2$ & $94.0 \pm 1.3$ \\
& 7 & $15.0 \pm 2.3$ & $13.7 \pm 0.2$ & $95.6 \pm 1.6$ \\
& 4 & $25.0 \pm 3.8$ & $26.3 \pm 0.3$ & $105 \pm 1$ \\
4 & $30.0 \pm 4.5$ & $31.4 \pm 0.3$ & $126 \pm 1$ \\
& 2 & $50.0 \pm 5.0$ & $52.0 \pm 0.5$ & $104 \pm 1$ \\
& 2 & $75.0 \pm 7.5$ & $74.1 \pm 0.7$ & $148 \pm 1$ \\
& 1 & $100 \pm 10$ & $107.8 \pm 1.5$ & $107.8 \pm 1.5$ \\
& 1 & $125 \pm 13$ & $132.7 \pm 1.3$ & $132.7 \pm 1.3$ \\
& 1 & $150 \pm 15$ & $152.3 \pm 0.2$ & $152.3 \pm 0.2$ \\
& 1 & $200 \pm 20$ & $210.2 \pm 0.4$ & $210.2 \pm 0.4$ \\
\hline \hline
\end{tabular}

tively. An example could thus be $2 \times$ Ta50Air 1000 , corresponding to two Ta foils, each $50 \mu \mathrm{m}$ thick, separated by $1 \mathrm{~mm}$ air.

There were some targets, which differed considerably from the intent of having same total thickness-viz. $4 \times$ Ta30Air1000, $2 \times$ Ta75Air1000, $1 \times$ Ta125, $1 \times$ $\mathrm{Ta} 150$, and $1 \times \mathrm{Ta} 200$. There were also some smaller discrepancies in $N \times\langle\delta t\rangle / X_{0}$ due to supplier's tolerances (cf. Table I).

The target assemblies were each held together by two brass end cap rings, which would be screwed tightly together. A drawing, showing an exploded view of the elements of a target assembly with $N=5$ layers, is shown in Fig. 3. The many targets were mounted at 16 positions forming a concentric circle of $200 \mathrm{~mm}$ radius on a target wheel, which allowed a remote-controlled target change by an azimuthal turn of the wheel. The angular position could be set and read back with a precision of a thousandth of a

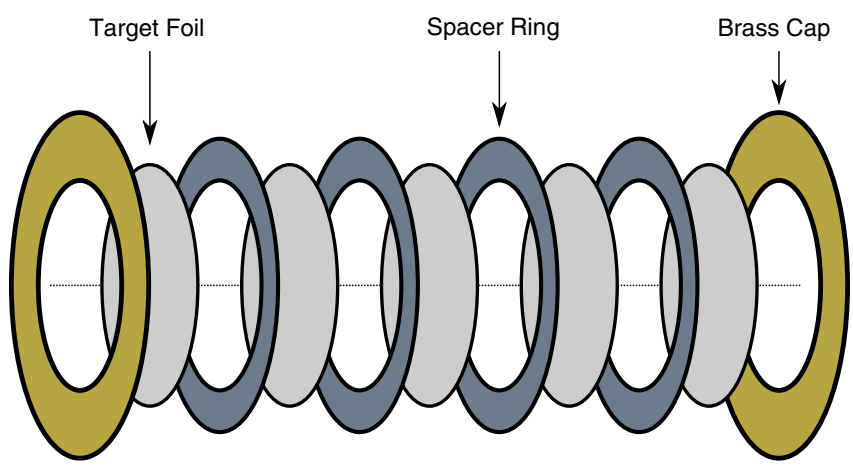

FIG. 3 (color online). Exploded view of a $N=5$ target. The dotted line illustrates beam $z$ axis.
TABLE II. Nominal and calculated electron beam energies at the SPS with the optics used.

\begin{tabular}{lc}
\hline \hline$E_{\text {Nom }}[\mathrm{GeV}]$ & $E_{\text {Calc }}[\mathrm{GeV}]$ \\
\hline 30 & 29.999 \\
60 & 59.983 \\
90 & 89.915 \\
120 & 119.731 \\
150 & 149.053 \\
180 & 178.650 \\
210 & 207.519 \\
\hline \hline
\end{tabular}

degree corresponding to $3.5 \mu \mathrm{m}$ arc length. The target wheel was placed about $10 \mathrm{~cm}$ from $\overline{\mathrm{S} 3}$.

\section{B. Calorimeters}

During the experiments two electromagnetic calorimeters were used-a Lead Glass (LG) detector of dimensions $90 \times 90 \mathrm{~mm}^{2}$ transverse area and $25 X_{0}$ long and a bismuth germanate (BGO) $\left[\left(\mathrm{Bi}_{2} \mathrm{O}_{3}\right)_{2}\left(\mathrm{GeO}_{2}\right)_{3}\right]$ detector of dimensions $\varnothing 75 \mathrm{~mm} \times 200 \mathrm{~mm}\left(18 X_{0}\right)$.

\section{Lead Glass}

The LG detector was calibrated using straight beams from the CERN SPS. The calorimeter was found to be very linear and exhibit acceptable resolution. The energies of the beams used for calibration and the experiments can be seen in Table II. Optimized beams were found and the beam elements were left untouched during each experiment. The TSF experiment was performed with the $149 \mathrm{GeV}$ beam, the LPM, and sandwich experiments, both with $207 \mathrm{GeV}$ electrons. Looking closer at the $149 \mathrm{GeV}$ calibration data for the LG, it was also found that this beam contained $\simeq 10 \%$ nonradiating particles-

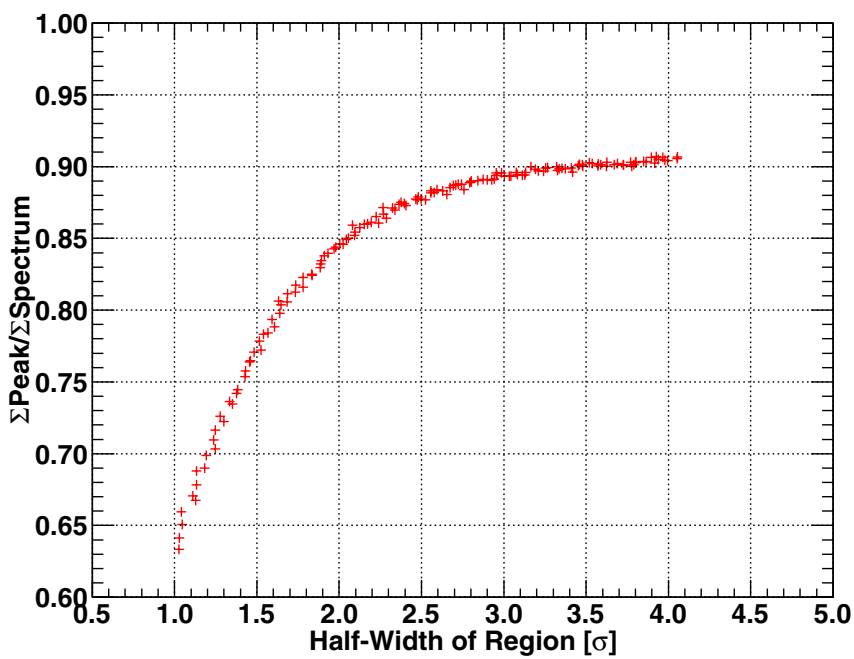

FIG. 4 (color online). Relative area of the photo peak as a function the half-width of the selected region in units of $\sigma$ of the photo peak. 
primarily $\mu^{-}$and $\pi^{-}$. This was found by comparing the counts in the photo peak to the total number of counts in the spectrum-cf. Fig. 4. Presence of nonradiating particles in the beam will make the number of triggers larger than the available number of electrons, thus affecting the normalization of our data. Our setup rendered no particle identification possible, since the particles reaching our setup all have the same momentum. A correction for the beam contamination is explained in Sec. IV B.

\section{2. $B G O$}

The BGO was partly calibrated at the Aarhus Storage Ring Denmark (ASTRID) since only high energy beams $(E \gtrsim 10 \mathrm{GeV})$ are feasible at the CERN SPS. In Aarhus,
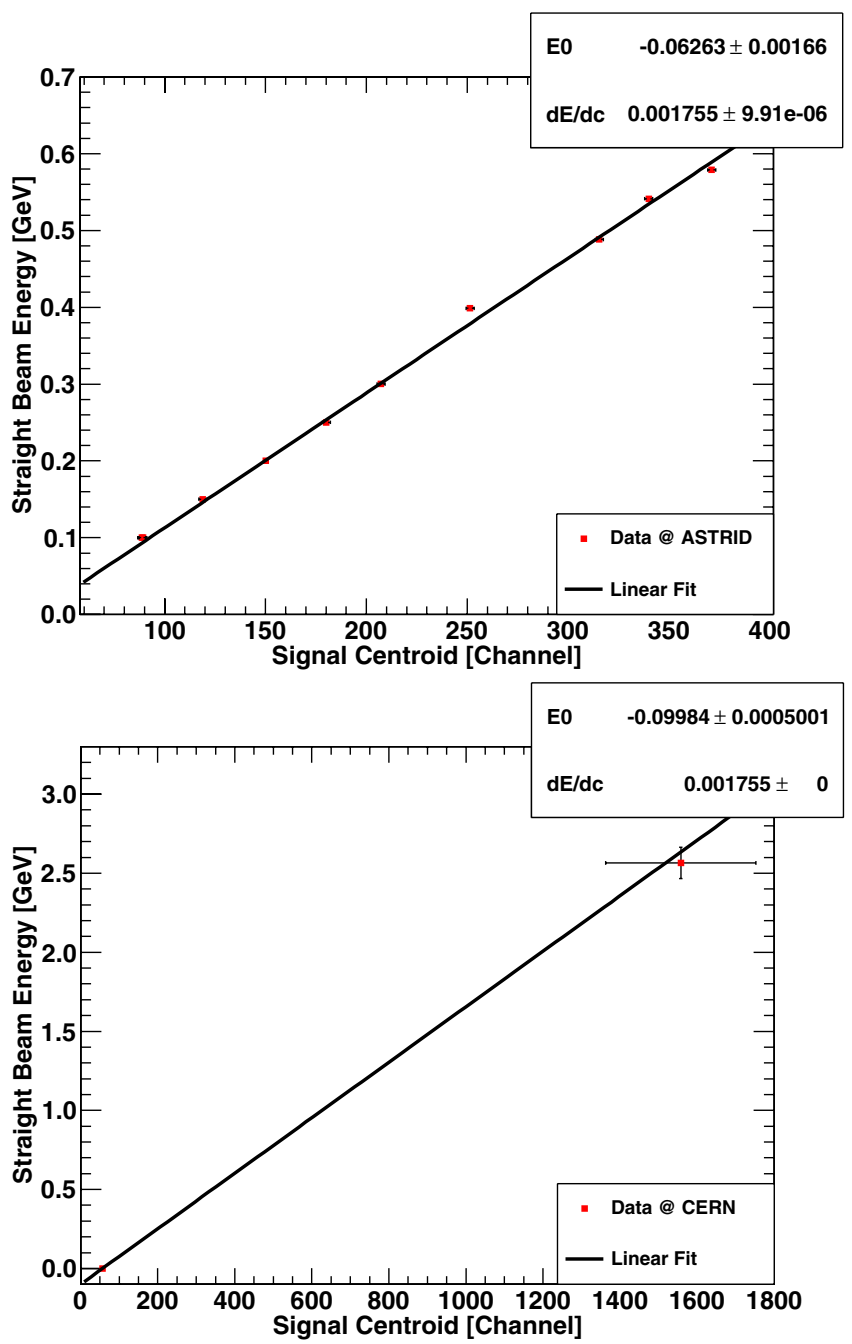

FIG. 5 (color online). BGO calibrations: (a) The calibration at Aarhus. Data points are shown with error bars representing statistical errors only. (b) The calibration at CERN. The error bars of the data point obtained through a tagged electron beam is relatively large due to a momentum spread of the accepted beam. Nevertheless, the ASTRID calibration is well verified only with a slight energy shift. the BGO was checked for linearity with the exact same electronics and settings utilized later at CERN. Results from the ASTRID calibration are shown in Fig. 5(a). With the reduced high voltage (HV) of $405 \mathrm{~V}$-as opposed to supplier's (Scionix) recommendations of 700-1100 Von the XP3330 Photomultiplier Tube (PMT), the BGO could detect photons of energies up to $\simeq 3 \mathrm{GeV}$. With many different, well-defined electron beam energies, the BGO was found to exhibit linearity despite the fact that it was operated well below the recommended HV. Using the same equipment and settings later at CERN, we trust the slope of the calibration to persist, but the baseline offset of the BGO signals could have been slightly different. To check this, we performed a calibration verification using a deflected electron beam having passed through a $10 \% X_{0}$ $\mathrm{Cu}$ radiator. This gave one point at $2.57 \mathrm{GeV}$ - determined by the chosen geometry and the well-known integrated field in B16-and the pedestal gave another point. A linear fit to these points can be found in Fig. 5(b). The slope of the fit is fixed to the value obtained from the Aarhus data. Notice the CERN calibration is shifted $\simeq-37(2) \mathrm{MeV}$ relative to the ASTRID one. The CERN calibration is used for the data analysis.

Not only is the BGO very linear despite the low HV, but the ASTRID calibration data is also in good accordance with Geant4 simulations at all calibration energies-an example is shown in Fig. 6. The simulations include all relevant electromagnetic processes, BGO crystal geometry and resolution, electron beam size, but not PMT resolution nor amplification. It should be noted, therefore, that the simulation showing the electron's energy deposit has been shifted in order to have the maximum at the beam's energy, but that the shape is unaltered.

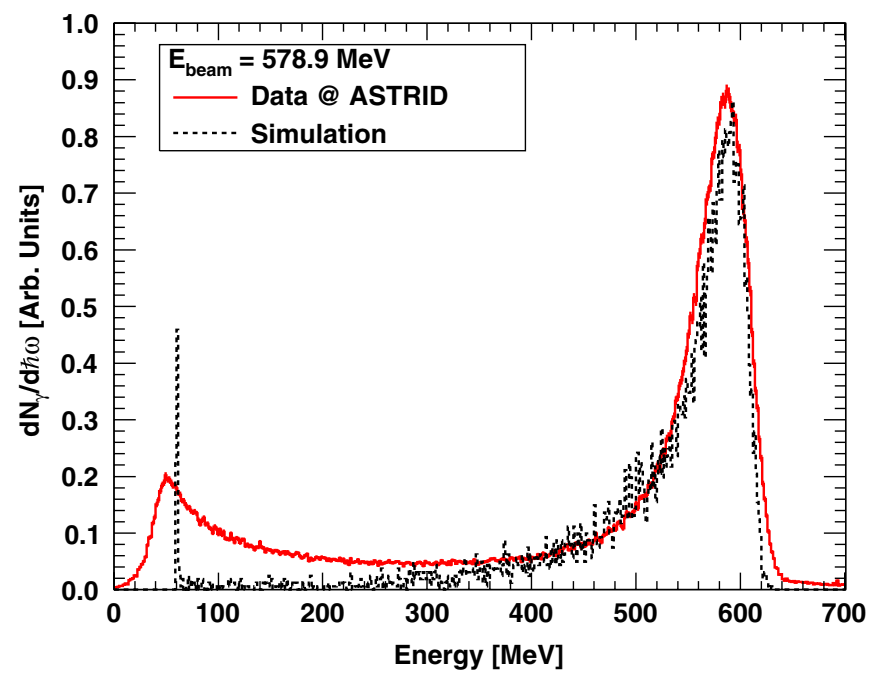

FIG. 6 (color online). Comparison between $e^{-}$BGO calibration data obtained at ASTRID (full, red) and Geant4 simulation (dotted, black). 


\section{ANALYSIS}

\section{A. BGO background}

The Synchrotron Radiation (SR) contribution caused by B16 is characterized by the critical energy $\hbar \omega_{c}=$ $3 \gamma^{3} e B \hbar / 2 p$. The maximum field in the magnet is $\simeq 2 \mathrm{~T}$ at which $\hbar \omega_{c}=30 \mathrm{MeV}(57 \mathrm{MeV})$ for $149 \mathrm{GeV}$ $(207 \mathrm{GeV})$ electrons. Beyond roughly this energy, the spectrum falls off exponentially. The resulting spectrum is a convolution of the SR contribution and the bremsstrahlung distribution. To deconvolute the spectrum and retain good statistics can be difficult, when it is heavily influenced by SR. The background power spectra per triggering event $1 / N_{e} \times d N_{\gamma} / d \ln \hbar \omega$ can be seen in Fig. 7. The backgrounds clearly contain a structure which grows close to exponentially when going to lower photon energies. The onset of the structure scales roughly with $\omega_{c}^{207} / \omega_{c}^{149}=$ 1.94. The lower photon energy detection limit is chosen as this onset of $\mathrm{SR}, \simeq 200 \mathrm{MeV}(\simeq 300 \mathrm{MeV})$ at $E=$ $149 \mathrm{GeV}(207 \mathrm{GeV})$. The backgrounds per electron reach the same level at higher photon energies in accordance with $\mathrm{BH}$, Eq. (1)-a reassuring feature. At lower energies the longitudinal density effect will set in from $\hbar \omega \lesssim \hbar \omega_{\mathrm{LD}}=$ 21.5 (30.0) MeV at $E=149 \mathrm{GeV}(207 \mathrm{GeV})$. Although the transition radiation yield is augmented by using structured targets, this effect shares the upper threshold of $\gamma \hbar \omega_{p}$ with the longitudinal density effect and is thus also negligible at $\hbar \omega \gtrsim 100 \mathrm{MeV}$. We fit the Bethe-Heitler expression (1) to the $149 \mathrm{GeV}$ background in the energy interval $0.3-3 \mathrm{GeV}$, where SR should be negligible. In this manner, the background is found to correspond to the radiation from a $2.06 \% X_{0}$ radiator - the fit is shown with a red, solid line in Fig. 7. With such low energy bremsstrahlung photons from a high energy beam, the LPM suppression and pile-up effects could have set in, subsequently rendering expres-

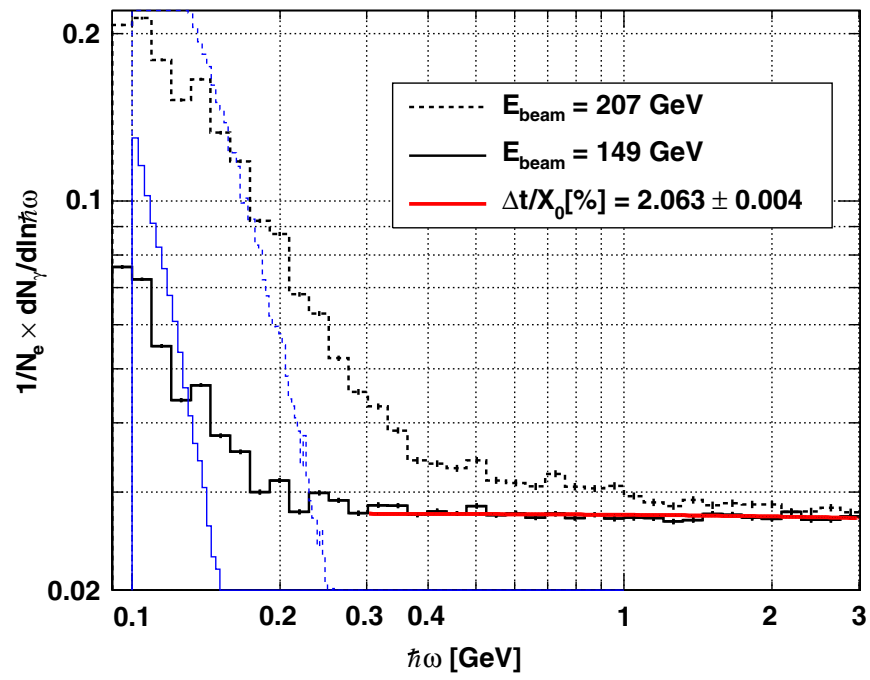

FIG. 7 (color online). BGO backgrounds: blue lines are Geant 4 simulations of synchrotron radiation generated in B16 at the two beam energies. sion (1) somewhat inappropriate in this region. Nevertheless, this level is in good accordance with backgrounds found in previous experiments [20]. Part of this is due to the scintillators before B16 (S1 and S2) which inevitably contribute to the photon background, as their combined thickness corresponds to $\simeq 0.9 \% X_{0}$.

\section{B. BGO efficiency}

The BGO is vital in our experiment to detect photons of energies down to $\simeq 200 \mathrm{MeV}$. During the preliminary tests of the BGO (i.e. the ASTRID calibration), it was found to be well suited for this task. Nevertheless, we have found on several occasions that running this relatively low energy calorimeter in the high energy experiment area at CERN is not an easy task.

The detector was subjected to photons of energies up to the electron beam's energy of several hundreds of GeV. Such hard radiation is believed not only to cause a deviation from linearity by heavy leakage but also to create a massive photon shower in the BGO which completely drains the PMT, subsequently leaving the detector inefficient for some time. An examination of this effect was attempted by setting up a Pattern Bit (PAT) in the CAMAC data acquisition system, which would mark the events following up to $200 \mu$ s after a very high pulse from the PMT-the logics is sketched in Fig. 8(a). In this way, a no high pulse (NHP) condition can be set in the offline analysis. Figure 8(b) shows raw power spectra (i.e. no background subtraction) that are normalized to number of triggers in the respective spectra. Of special interest in this figure is a comparison of the red, open (no pattern condition), and filled squares (NHP condition). Applying the condition clearly has an effect on the level of the power spectrum. The cause of inefficiency thus identified, we corrected for it as described in the following.

Using a refined version of the proper Geant 4 implementation of bremsstrahlung (improved Bethe-Heitler with electronic contribution and Coulomb correction [[14], Eq. (3.83)] and Migdal's LPM formulation [25]) described in [35] a simulation for the $80 \times \mathrm{A} 125 \mathrm{~A}$ ir 1000 target was performed. Since each individual Al foil is significantly thinner than the multiple scattering length of the material $\ell_{\gamma}=\frac{\alpha X_{0}}{4 \pi}=51.7 \mu \mathrm{m}$ (cf. p. 3), this target should belong to the $\mathrm{BH}$ regime and be a simple, good spectrum reference. The simulation includes solid angle of the detector, pair production in air and foil, multiphoton emission, and other relevant, physical processes.

In Fig. 9, the normalized power spectrum is shown in a logarithmically binned histogram. This is found by subtracting the background - which is shown in Fig. 8(b) as black circles-from the target measurement, each normalized to the respective number of triggering events $\left(N_{e}\right)$. The resulting spectrum is then normalized to the target thickness in units of radiation lengths $\left(\Delta t / X_{0}\right)$, cf. Table I. The uncorrected power spectrum (red squares) is seen to be 
(a)

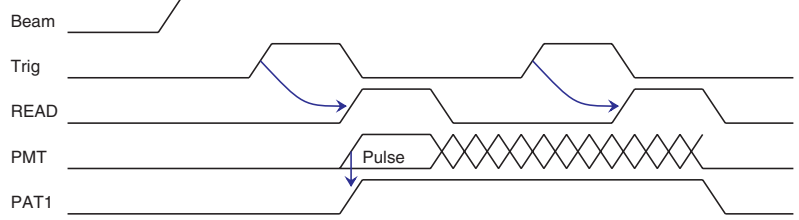

(b)

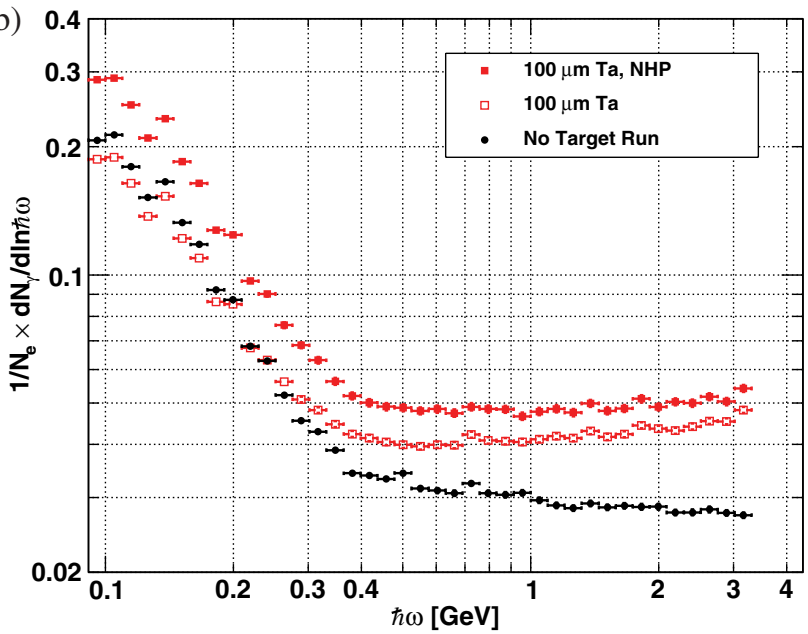

FIG. 8 (color online). NHP Analysis: rejecting events following large pulses clearly changes the power spectrum. (a) NHP Logics: the first event (Trig) is a large pulse, which causes the analog PMT signal to enter an undefined state. PAT1 tags the possible, subsequent events that are considered flawed. (b) Results of applying the NHP condition.

some $\simeq 20 \%-40 \%$ below the GEANT3 simulation. To summarize, this deficiency is believed to originate from two contributions

(1) As seen in Fig. 4, the number of radiating particles $\left(e^{-}\right)$in the beam is $\simeq 90 \%$ of the number of events $\left(N_{e}\right)$. By normalizing to $N_{e}$ the overall norm of the

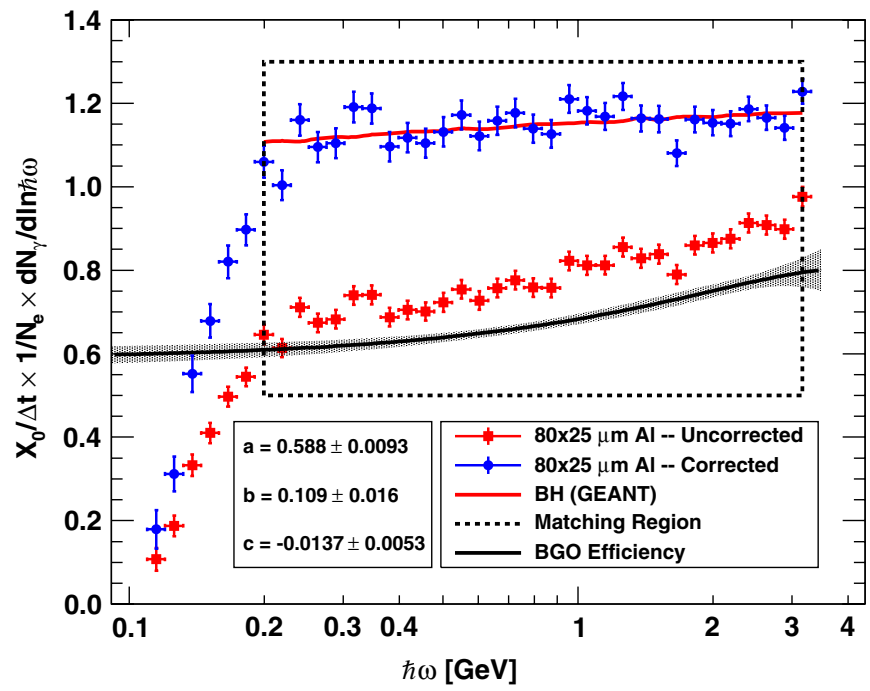

FIG. 9 (color online). BGO efficiency correction: a relatively flat polynomial can bring the data points in good agreement with simulations above $0.2 \mathrm{GeV}$. power spectrum is erroneously lowered by this factor.

(2) Hard radiation from the high energy beam is believed to drain the PMT.

The BGO efficiency and beam purity is presumed to be well described by a quadratic polynomial of photon energy

$$
\mathcal{E}=a+b \times \hbar \omega / \mathrm{GeV}+c \times(\hbar \omega / \mathrm{GeV})^{2},
$$

and the data divided by such a model are matched to the GEANT3 simulation within the photon energy region marked in Fig. 9 by minimizing an $\chi^{2}$ statistic. Notice how the spectrum dives towards zero when $\hbar \omega \lesssim$ $200 \mathrm{MeV}$, indicating an increasing SR contamination below this point. The obtained model parameters $a-c$ along with a plot of the fitted efficiency and the $95 \%$ confidence interval from the fit can also be seen in this figure, where the data and $\mathcal{E}$ share ordinate. The BGO temperature was monitored during the experiment and only very small deviations were found ( $\lesssim 1 \mathrm{~K}$ ), and thus not corrected for.

\section{RESULTS}

\section{A. Thin foils}

The event-based data is projected onto logarithmically binned photon energy histograms-one for each targetwith left edge of the $i$ th bin following $10 \mathrm{MeV} \times$ $10^{(i-1) / 25}$, i.e. 25 bins/decade. The resulting power spectra are normalized to the number of trigger events, the background is subtracted. The result is efficiency-corrected and normalized to the measured value of $\Delta t / X_{0}$. The spectra can be seen in Figs. 10(a)-10(d). Notice that they tend to mutually converge at large photon energies. They clearly have different shapes, although the targets all have approximately the same total thickness in units of radiation lengths $\Delta t / X_{0} \simeq 2.44 \% \sim 1 \times$ Ta100. The power spectra almost all tend to have a kink around $\hbar \omega \simeq 0.3 \mathrm{GeV}$, below which the data points seem to be systematically lower than the theory curves. This could be caused by a still present contamination of SR, which would be more predominant in the background spectrum, hence cause an overestimation of the background. In the Figs. 11(a), 11(b), 11(c), 11(d), 11(e), 11(f), 11(g), 11(h), 11(i), and 11(j), the power spectrum level is plotted as a function of foil thickness in units of $X_{0}$ for each relevant photon energy bin center. In essence, the figures show the probability of radiation emission per target atom encounter, as a function of the number of scattering events. Initially, for target thicknesses below $l_{\gamma}$, the radiation probability per encounter is equal to the $\mathrm{BH}$ value because a scattering event is unlikely to be significantly affected by the distortion of the Coulomb field of the electron resulting from a previous event. Then, as the thickness increases, the emission probability per encounter drops drastically by up to a factor 3 for photon energies of $\simeq 0.3 \mathrm{GeV}$ - the distorted Coulomb field of the electron 

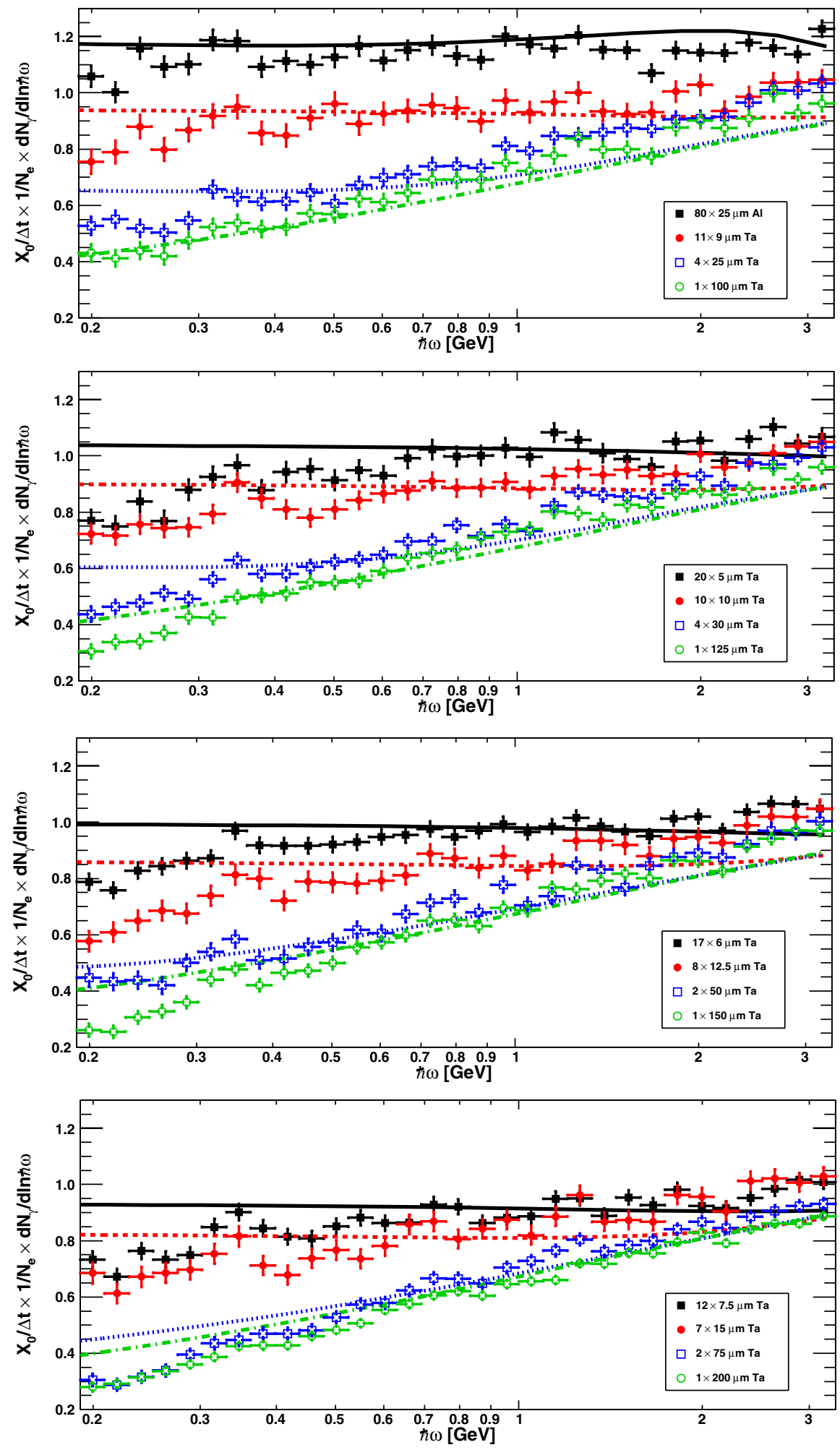

FIG. 10 (color online). The data points show the number of photons emitted $N_{\gamma}$ in a logarithmically binned interval of photon energies (25 bins/decade) for various targets of the type $N \times A \delta t A i r 1000$. The resulting power spectrum is corrected with the BGO efficiency and normalized to both the number of events $N_{e}$ and the combined target thickness in units of the material's radiation length $\Delta t / X_{0}$. The lines show calculations of the power spectrum of each target using the Blankenbecler-Drell formalism [1]. As commented elsewhere, the calculations have been corrected by a factor 0.85 to take multiphoton emission and pair production into account. 
(a)

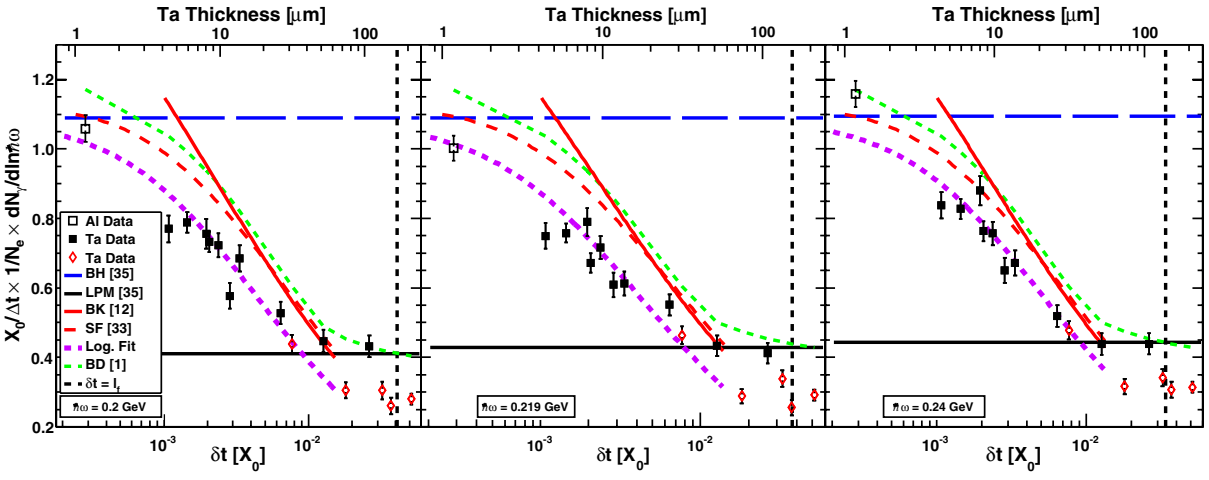

(b)

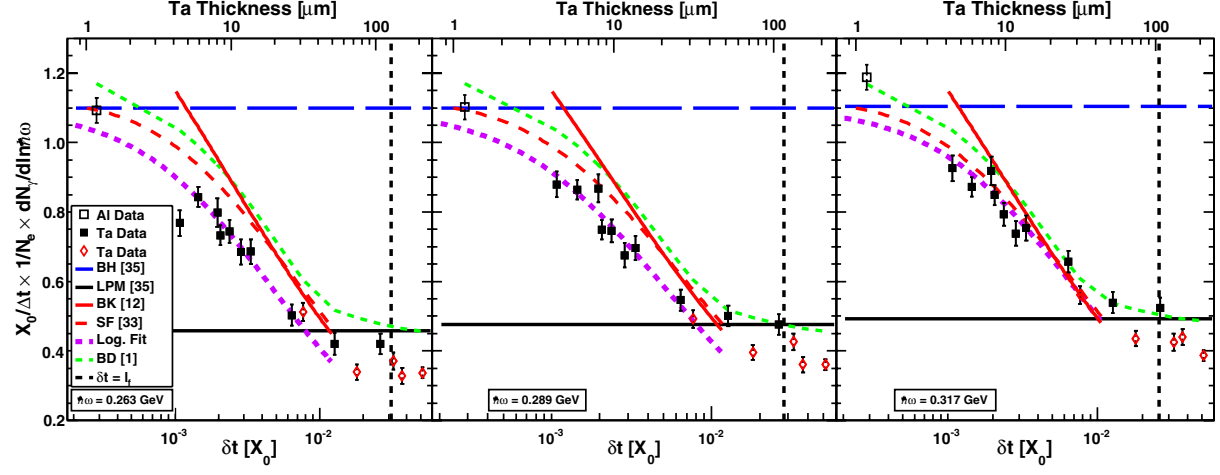

(c)

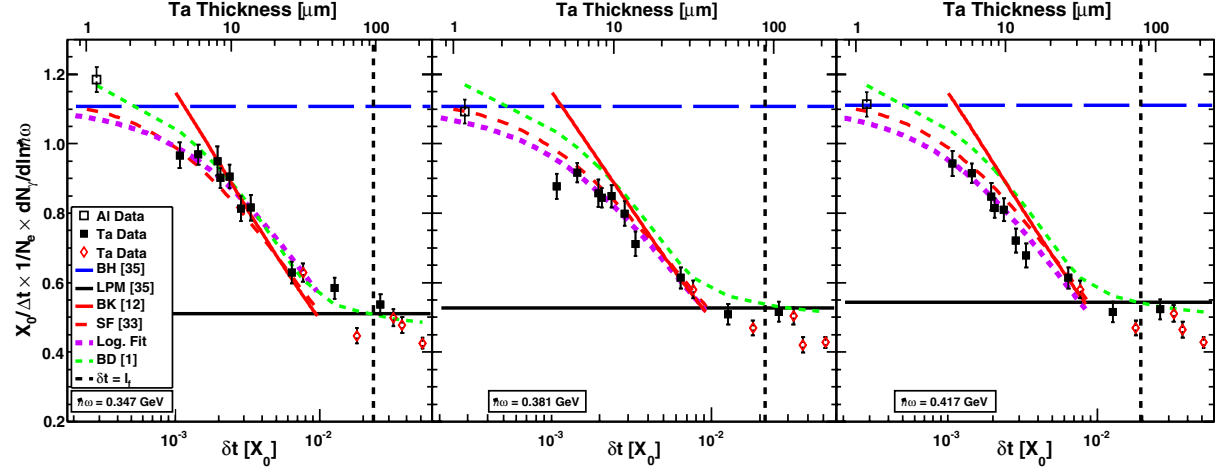

(d)

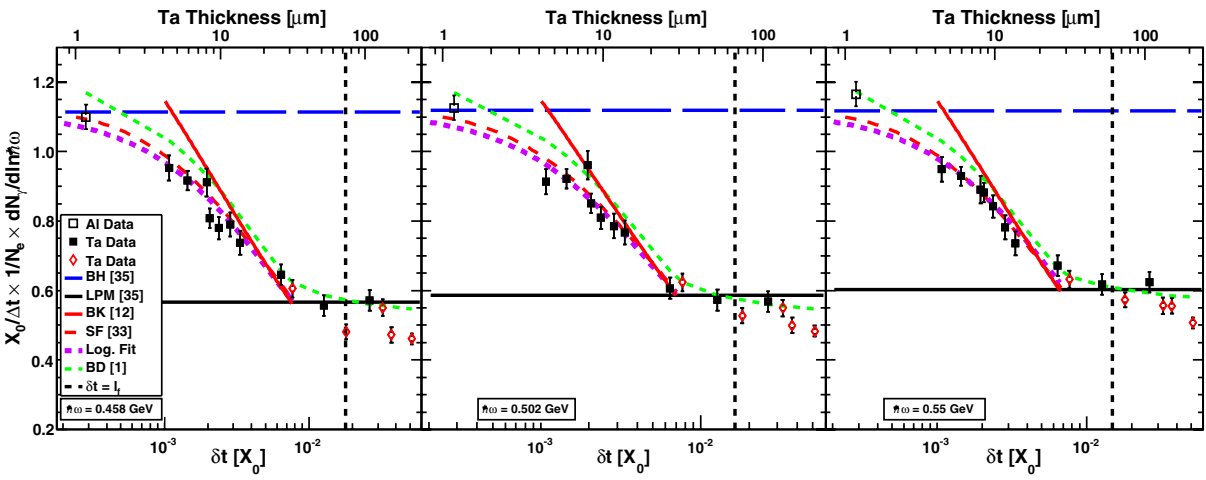

FIG. 11(a-d) (color online). The normalized power spectrum level as a function of the independent foil thickness $\left(\langle\delta t\rangle\right.$ in units of $X_{0}$ on lower scale, equivalent tantalum foil thickness on upper scale) in the different photon energy bins with the centroid value given. With squares and diamonds (with error bars denoting the statistical uncertainty only) are shown the measured values. The open, square data point at $\delta t=3 \times 10^{-4}$ is for the $80 \times 25 \mu \mathrm{m} \mathrm{Al}$ foil, while the open, diamond data points are for the targets with $\Delta t / X_{0} \gtrsim 1.2 \times 2.44 \%$-i.e. not quite comparable to $100 \mu \mathrm{m}$ Ta. The horizontal lines are the GEANT3 simulated values for $100 \mu \mathrm{m}$ Ta according to Bethe-Heitler (long-dashed line) and LPM theory (full line). The falling curves are calculations of the logarithmic dependence of intensity on thickness (which decreases when divided by the thickness) following [33] (long-dashed), [12] (full), [1] (dashed), and the function in Eq. (7) (dotted). The vertical dashed line shows the thickness corresponding to the formation length, i.e. the critical thickness for the given photon energy. 
(e)

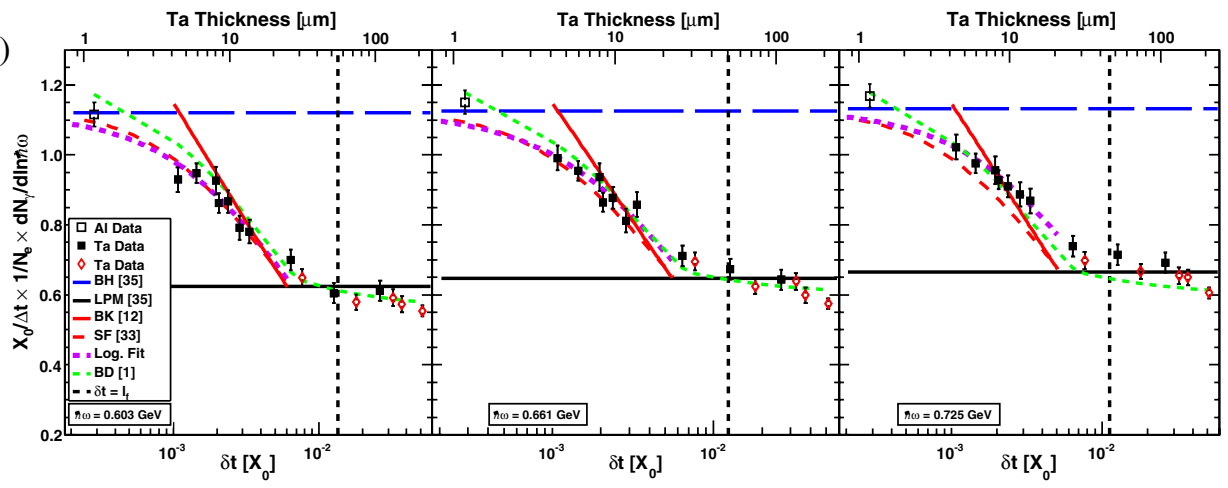

(f)

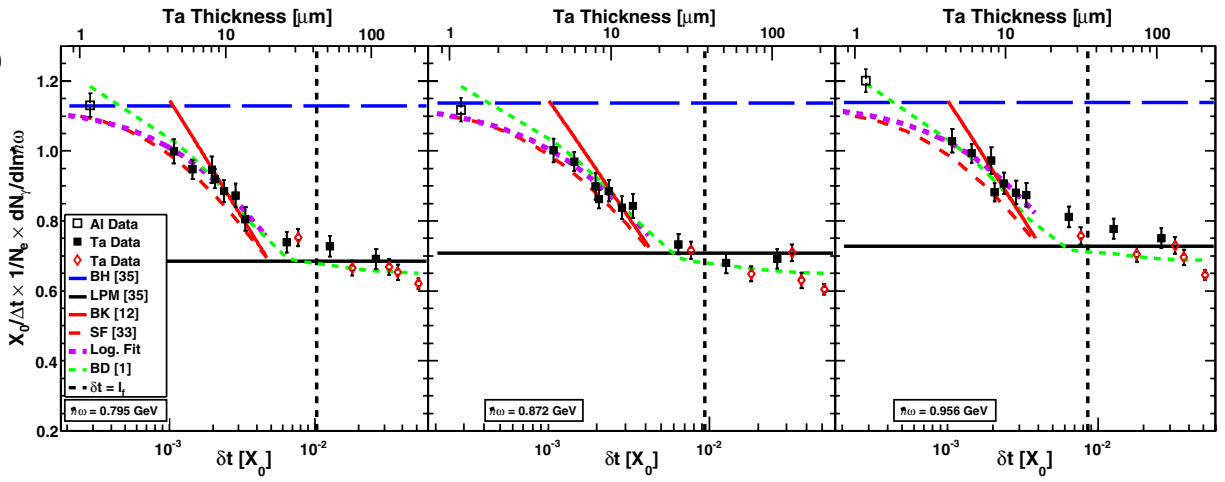

(g)

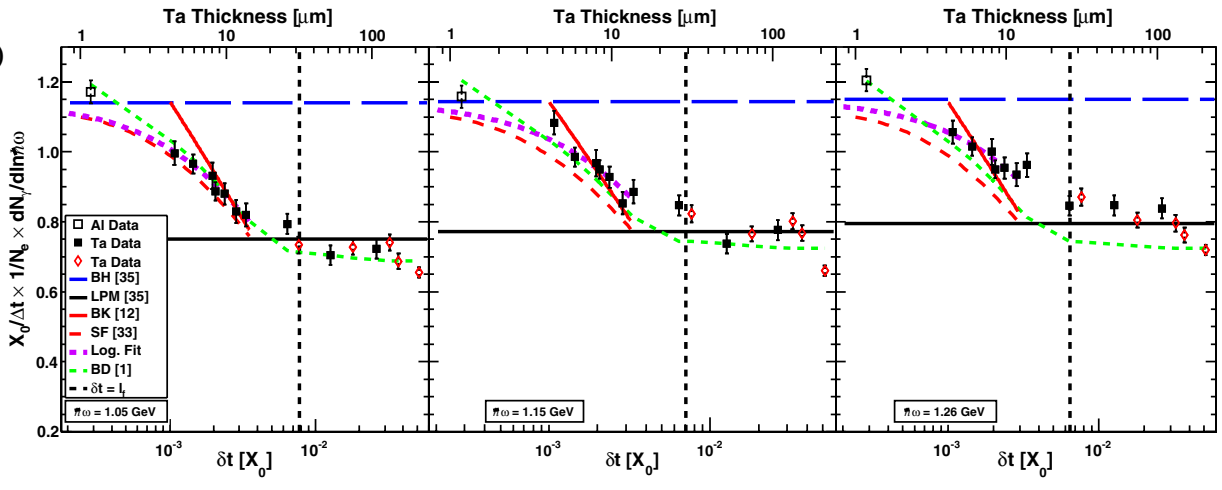

(h)

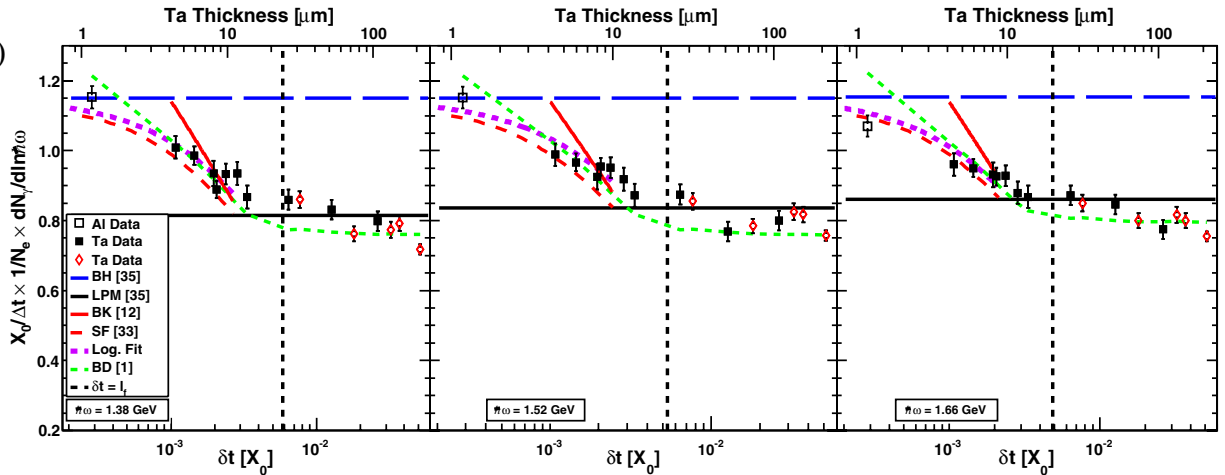

FIG. 11(e-h) (color online). The normalized power spectrum level as a function of the independent foil thickness $\left(\langle\delta t\rangle\right.$ in units of $X_{0}$ on lower scale, equivalent tantalum foil thickness on upper scale) in the different photon energy bins with the centroid value given. With squares and diamonds (with error bars denoting the statistical uncertainty only) are shown the measured values. The open, square data point at $\delta t=3 \times 10^{-4}$ is for the $80 \times 25 \mu \mathrm{m} \mathrm{Al}$ foil, while the open, diamond data points are for the targets with $\Delta t / X_{0} \geq 1.2 \times 2.44 \%$-i.e. not quite comparable to $100 \mu \mathrm{m} \mathrm{Ta}$. The horizontal lines are the GEANT3 simulated values for $100 \mu \mathrm{m}$ Ta according to Bethe-Heitler (long-dashed line) and LPM theory (full line). The falling curves are calculations of the logarithmic dependence of intensity on thickness (which decreases when divided by the thickness) following [33] (long-dashed), [12] (full), [1] (dashed), and the function in Eq. (7) (dotted). The vertical dashed line shows the thickness corresponding to the formation length, i.e. the critical thickness for the given photon energy. 
(i)

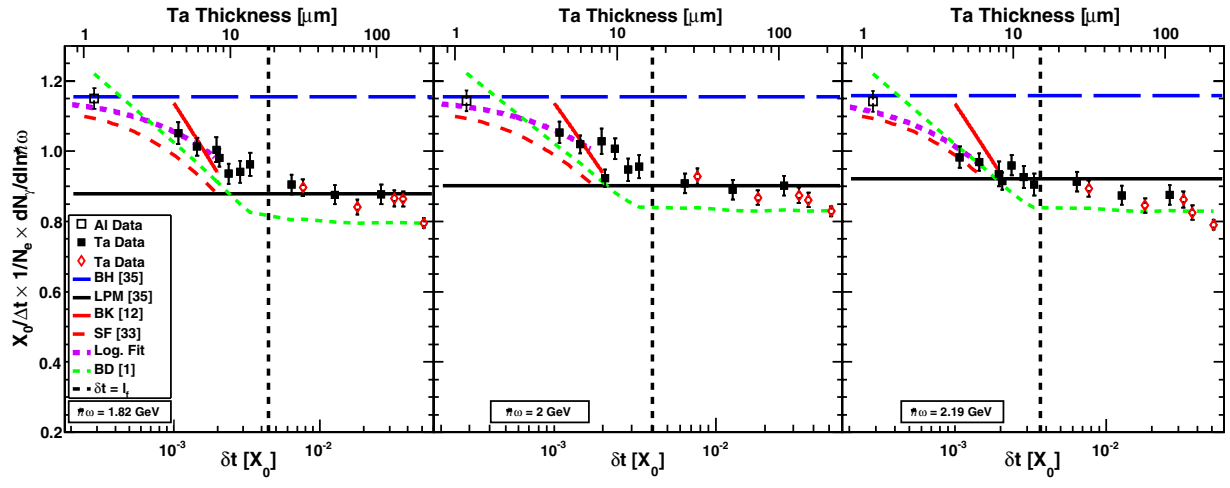

(j)

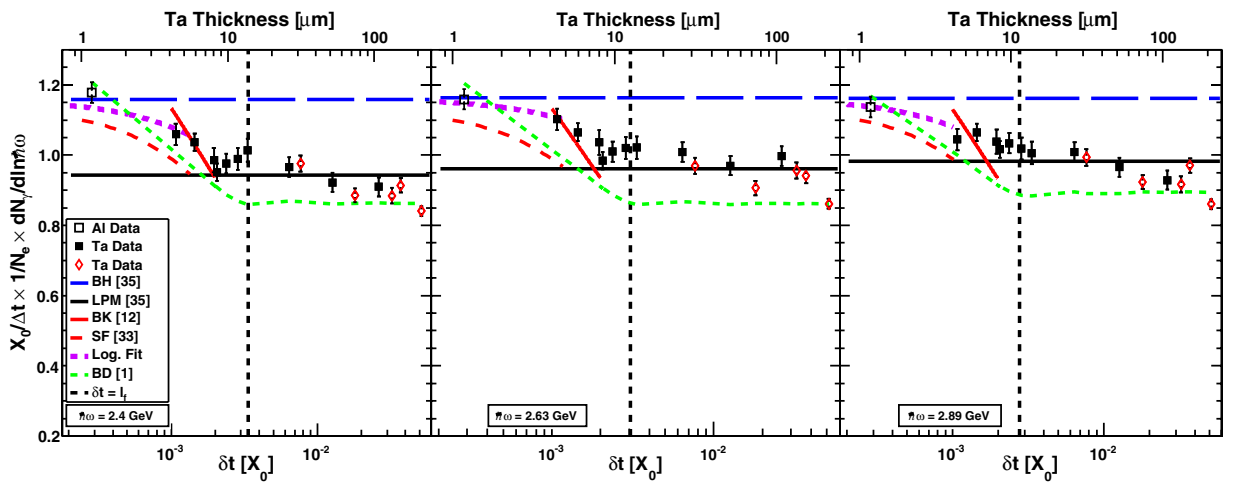

FIG. 11(i,j) (color online). The normalized power spectrum level as a function of the independent foil thickness ( $\langle\delta t\rangle$ in units of $X_{0}$ on lower scale, equivalent tantalum foil thickness on upper scale) in the different photon energy bins with the centroid value given. With squares and diamonds (with error bars denoting the statistical uncertainty only) are shown the measured values. The open, square data point at $\delta t=3 \times 10^{-4}$ is for the $80 \times 25 \mu \mathrm{m} \mathrm{Al}$ foil, while the open, diamond data points are for the targets with $\Delta t / X_{0} \gtrsim 1.2 \times 2.44 \%$-i.e. not quite comparable to $100 \mu \mathrm{m}$ Ta. The horizontal lines are the GEANT3 simulated values for $100 \mu \mathrm{m}$ Ta according to Bethe-Heitler (long-dashed line) and LPM theory (full line). The falling curves are calculations of the logarithmic dependence of intensity on thickness (which decreases when divided by the thickness) following [33] (long-dashed), [12] (full), [1] (dashed), and the function in Eq. (7) (dotted). The vertical dashed line shows the thickness corresponding to the formation length, i.e. the critical thickness for the given photon energy.

influences radiation emission in the consecutive scattering events. Then eventually a level determined by the LPM mechanism is reached, where multiple scattering dominates the process and essentially all events are influenced by the reduction. As the photon energy increases, the formation length decreases, and more target thicknesses enter the LPM regime, as $\ell_{\gamma} \lesssim \ell_{\mathrm{f}} \lesssim \delta t$.

The data is compared with the GEANT3 simulation of the BH and LPM level (shown as horizontal lines) for $1 \times$ Ta100 at the corresponding value of $\hbar \omega$. It is important to note that pile-up (multiphoton) effects will to first order depend on the total target thickness $\left(\Delta t / X_{0}\right)-\operatorname{not}\langle\delta t\rangle / X_{0}$, which is shown on the abscissa.

\section{Comparison with theory}

Several theories have been numerically implemented: Baier and Katkov's (BK) Eq. (5.15) with (5.12) of [12] (red, full line), Shul'ga and Fomin's (SF) Eq. (4.9) [33] (red, dashed line), and Blankenbecler and Drell (BD) [11] (green, dotted line). As demonstrated in the latter reference, the BD formalism is well capable of calculating the modification of the $\mathrm{BH}$ spectrum at many different target thicknesses, whereas the other two focus on asymptotic expressions valid in certain thickness regimes.

The hitherto listed theories only consider single-photon emission, but multiphoton emission has a considerable effect on the photon spectrum, even at the thin foils we have considered experimentally, rendering the theories somewhat inappropriate. In a later paper, BK have described multiphoton effects for soft photons $(y \ll 1)$ in various foil thickness regimes [36]. The correction is in each case described by a multiplicative function $f(\Delta t, \hbar \omega)$, which is calculated from the single-photon emission probability. In Fig. 12, the multiphoton correction in the LPM regime is plotted [[36], Eq. (2.27)]. As can be seen here, the correction to their single-photon expression depends very much on the total target thickness. The correction is by nature also photon energy dependent. The correction for $100 \mu \mathrm{m}$ is seen to be some $85 \%-90 \%$ below the singlephoton spectrum in the shown photon energy range.

Although BK's theory [36] includes multiphoton effects, numerical implementation is nevertheless only feasible using asymptotic expressions in the various thickness re- 


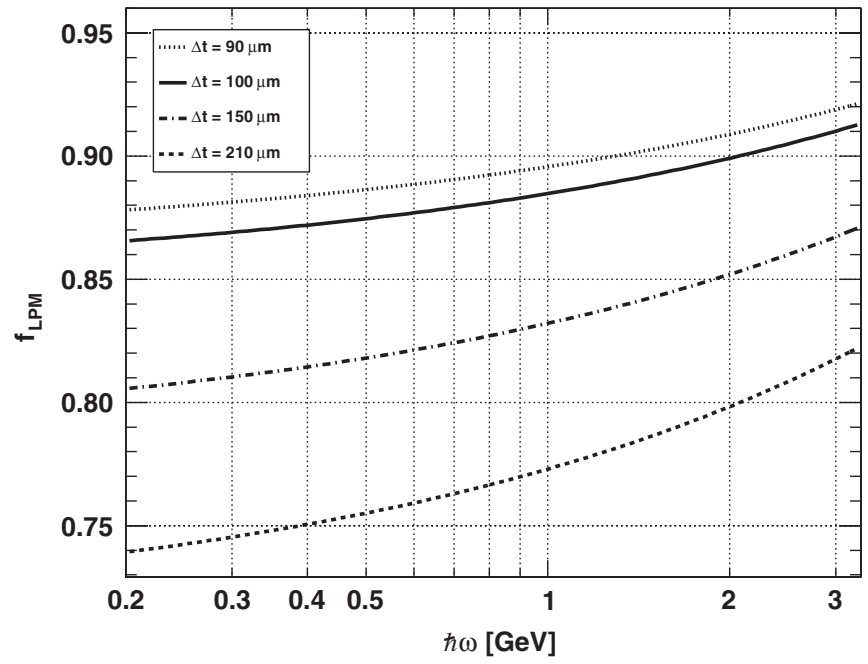

FIG. 12. Multiphoton correction factor for Ta foils of various total thickness $\Delta t$. These calculations presuppose a singlephoton spectrum based on the LPM effect.

gimes in which the correction factors are slightly different. The radiation spectrum will also be influenced by secondary pair production in the target. Any such pairs would not reach our calorimeters because of B16, leaving only the radiation. Pair production and multiphoton emission influences the radiation spectrum in similar degree due to the crossing symmetry of the two secondary processes. GEANT3 simulations have confirmed that the soft radiation spectrum will be additionally suppressed by subsequent pair production in the target-an effect that neither of the mentioned theories include.

We employ a simple correction of the single-photon theories of SF, BK, and BD by multiplying their level by a constant of $85 \%$-hence ignoring the dependence on $\hbar \omega$-which leaves the corrected theories in better accordance with the GEANT3 simulations. As seen in Fig. 12, we have with this simple correction neglected a small, relative difference $\simeq 8 \%$. The correction adds to the systematic uncertainty of the theoretical curves shown.

We stress that the measured data points have only been corrected by the BGO efficiency. The deviation from theory observed at large thicknesses is partly explained by multiphotons, which give a reduction of $13 \%-15 \%$ at $200 \mu \mathrm{m}$ thickness compared to $100 \mu \mathrm{m}$ for which simulations have been performed, cf. also Fig. 12. The previously mentioned Ta targets with a thickness deviating markedly from that of $1 \times \mathrm{Ta} 100\left(\Delta t / X_{0} \gtrsim 1.2 \times\right.$ $2.44 \%$ ) are explicitly marked in the figures. The numerous figures showing the thickness dependence of the power spectrum for fixed photon energy have been compiled into an animation which can be found online [37].

The simple, logarithmic model in Eq. (7) has been utilized to quantify the data's logarithmic resemblance. In each of the Figs. 11(a), 11(b), 11(c), 11(d), 11(e), 11(f), 11(g), 11(h), 11(i), and 11(j), a least-squares fit with the model has been performed. In each fit, the parameter $a$ is fixed to the GEANT3 simulated value of the BH level (also shown in the figures). Neither the model nor [33] include the LPM effect, and hence the fit is only performed to the data points with $\delta t$ below the crossing point of the LPM curve and the theoretical curve of SF [33]. The simple expression describes both the data and the SF calculation well. A fit to the latter yields $b=319 / X_{0}$. The values of $b$ extracted from the fit, shown in Fig. 13, are in fair agreement with the expected theoretical value and therefore almost the entire data set of 30 logarithmic bins in photon energy is well described by the same simple logarithmic function.

At the very lowest photon energies above the detection threshold, the data points in Fig. 11(a) are seen to be systematically lower than the theory curves, as previously noted in the power spectra, Figs. 10(a)-10(d). At $\hbar \omega \lesssim$ $0.3 \mathrm{GeV}$, this effect results in a systematically larger value of $b$. At $\hbar \omega \gtrsim 2.2 \mathrm{GeV}$ only one or two data points contribute to the fit making its credibility questionable. The discrepancy with theory at high photon energies may also be due to the total uncertainty in the detection efficiency, which we estimate to be about $5 \%$ in the interval $0.3-$ $2.2 \mathrm{GeV}$ (filled squares), i.e. there is a systematic uncertainty of about the same size as the statistical. Outside this range, the BGO calibration has a systematic uncertainty at least about twice as large (open squares). Only statistical errors are shown.

\section{B. Low Z LPM}

In Table III, the LPM thresholds $\hbar \omega_{\text {LPM }}$ are shown for carbon (rigid graphite), aluminum, and tantalum. As seen here, at least aluminum and tantalum should be within the LPM regime using a $207 \mathrm{GeV}$ beam. In the experiment, the LPM targets were mounted on the target wheel, and $N_{e} \simeq$ $1 \times 10^{6}$ events were registered for each of the three targets and the background.

The resulting normalized power spectra-with background subtracted properly (as outlined above)-are shown in Figs. 14(a)-14(c). The efficiency-corrected data is - at least in the cases of aluminum and tantalum-in remarkable agreement with the LPM simulations, giving good confidence in the efficiency correction. As for carbon, the LPM threshold is on the verge of the lower detection limit—clearly seen in the lowest energy point—and the statistics are too low to see a consistent tendency. There are

TABLE III. Radiation lengths [14], target thicknesses, and LPH photon energy thresholds for the three materials and beam energies used.

\begin{tabular}{lccc}
\hline \hline & $\mathrm{C}$ & $\mathrm{Al}$ & $\mathrm{Ta}$ \\
\hline$X_{0}[\mathrm{~mm}]$ & 245.4 & 88.97 & 4.094 \\
$\Delta t / X_{0}[\%]$ & $1.97(1)$ & $2.27(2)$ & $2.63(4)$ \\
$\hbar \omega_{\mathrm{LPM}}^{149}[\mathrm{GeV}]$ & 0.118 & 0.324 & 6.74 \\
$\hbar \omega_{\mathrm{LPM}}^{207}[\mathrm{GeV}]$ & 0.228 & 0.628 & 12.8 \\
\hline \hline
\end{tabular}




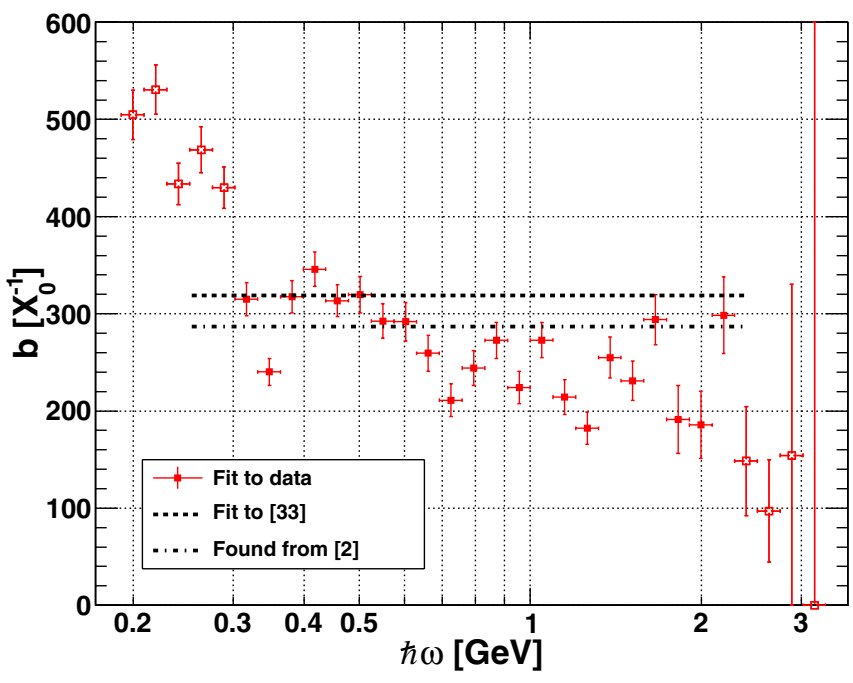

FIG. 13 (color online). The $b$ parameter extracted from leastsquares fits with the logarithmic function $a \ln (b \times\langle\delta t\rangle+$ $1) /(b \times\langle\delta t\rangle)$, as a function of photon energy. The horizontal lines show the value of $b$ estimated from [33] and the leading order value found from [2], Eq. (30), shown in Eq. (8). Systematic uncertainties are estimated to be $\simeq 5 \%$ (filled points) and $\simeq 10 \%$ (open points).

nevertheless no indications of discrepancies except at the lowest photon energy.

\section{Sandwich Effect}

A reference target $10 \times \mathrm{Ta} 10 \mathrm{Air} 1000$ and a sandwich target $10 \times$ Ta10Air90 were prepared using the scheme sketched in Fig. 3. The $\simeq 1 / 10$ spacing in the sandwich target was acquired using $\langle\delta g\rangle=88.1 \pm 0.7 \mu \mathrm{m}$ thick surface polished phosphor-bronze foils with a $\varnothing 18 \mathrm{~mm}$ hole. With this spacing, resonances between the foils should be possible for $\ell_{\mathrm{f}} \gtrsim \delta g \Rightarrow \hbar \omega \lesssim 0.736 \mathrm{GeV}$, cf. Equation (9). By measuring the sandwich target assembly's full thickness with a micrometer gauge and subtracting the known amount of material besides the spacer foils, we have estimated the actual mean spacing to $\langle\delta g\rangle=$ $91.2 \pm 0.7 \mu \mathrm{m}$, not far from the expected $88.1 \mu \mathrm{m}$. The $3.1 \mu \mathrm{m}$ extra material most likely stems from air between nonclose-fitting foils. Performing Monte Carlo simulations of targets consisting of the ten spacer foils $(\langle\delta g\rangle=88.1 \pm$ $0.7 \mu \mathrm{m})$ and extra space with mean thickness of $3.1 \pm \sigma$, we have estimated the probability of a given value of $\sigma$ and still being consistent with $\langle\delta g\rangle=91.2 \pm 0.7 \mu \mathrm{m}$. The result is roughly an exponential function $P(\sigma) \propto$ $\exp \left(-\sigma / \sigma_{\mathrm{MC}}\right)$ with $\sigma_{\mathrm{MC}}=(4.3 \pm 2.1) \mu \mathrm{m}$. Having a spread of several multiples of this parameter is consequently very unlikely. Thus, the spacing of foils is likely to be $91.2 \mu \mathrm{m}$ with a variation of $\lesssim 5 \%$. Measuring the small, actual spacings in the target assembly without affecting the result is very difficult and has not been attempted.
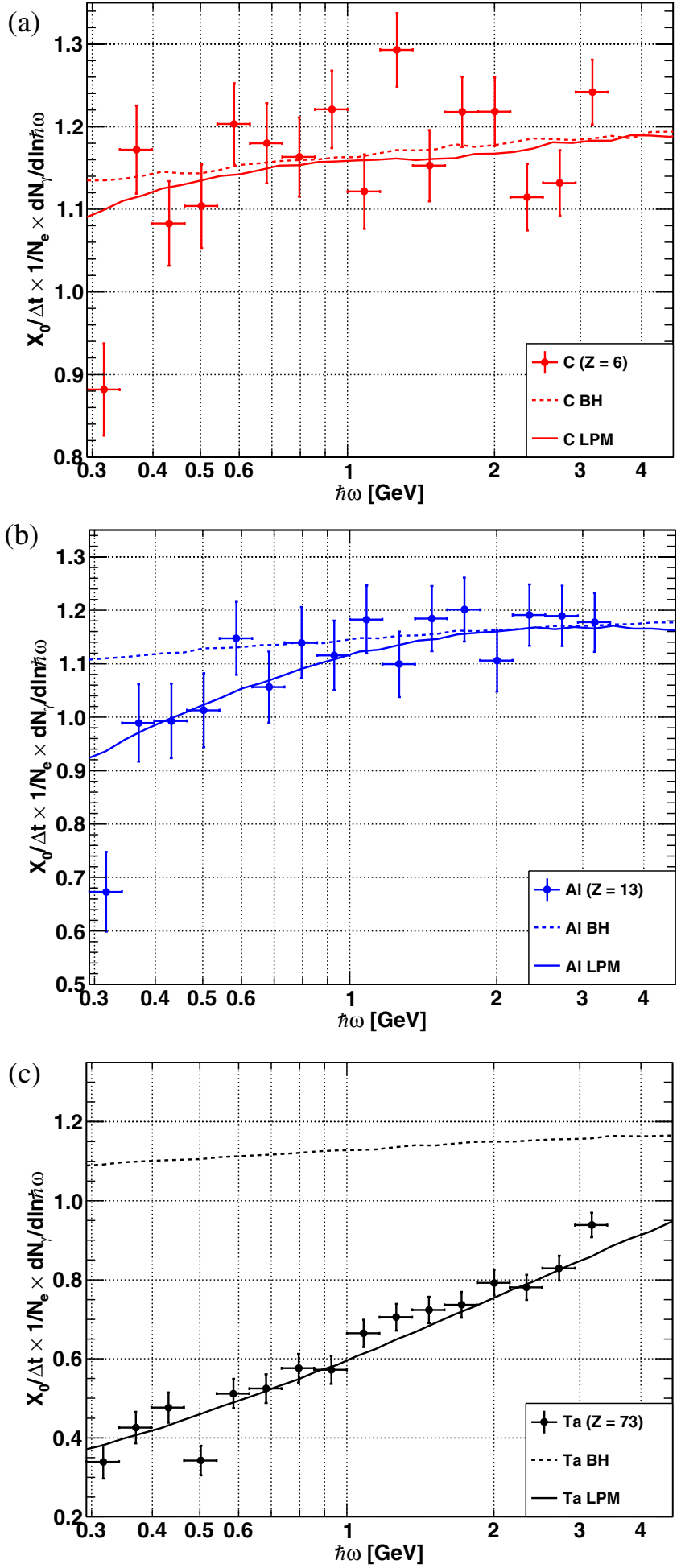

FIG. 14 (color online). Normalized power spectra for different materials at $E=207 \mathrm{GeV}$. The experimental data points have been scaled by the BGO efficiency shown in Fig. 9. The three spectra carry similar statistics but are shown with different ordinate scales. The dotted and full lines are the GEANT3 simulations of the Bethe-Heitler and LPM theory, respectively. 

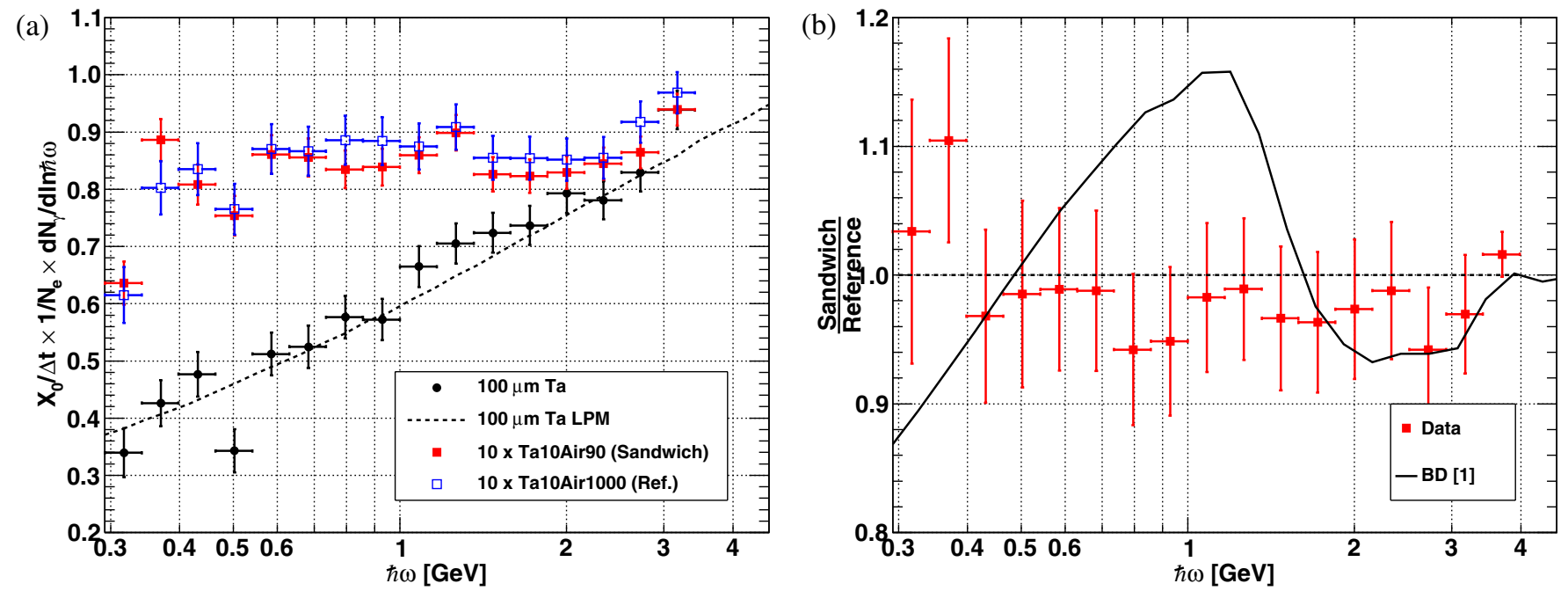

FIG. 15 (color online). Absolute and relative sandwich spectre measured at $E=207 \mathrm{GeV}$. The reference and sandwich target spectra are close to identical. (a) Normalized power spectra for the different foil types. The LPM data for the $1 \times 100 \mu \mathrm{m}$ Ta target is shown again for reference. (b) Ratio of measured power spectra, $(10 \times \mathrm{Ta} 10 \mathrm{Air} 90) /(10 \times \mathrm{Ta} 10 \mathrm{Air} 1000)$, and corresponding ratio of BD calculations [1].

The two sets of $10 \times 10 \mu \mathrm{m}$ Ta foils used in the assemblies of the TSF and sandwich targets came from two different supplier batches and were found to be slightly dissimilar. The combined thickness of the reference target foils was $97.4 \pm 1.1 \mu \mathrm{m}$, whereas it was $118 \pm 1 \mu \mathrm{m}$ for the sandwich target. This difference in target thickness does not introduce any serious discrepancy due to multiphoton effects. The normalized power spectra are shown in Fig. 15(a). Both multifoil targets (square points) exhibit a distinctive excess of radiation at lower energy when compared to the single $100 \mu \mathrm{m}$ Ta foil, but there are only very small differences between the two. To magnify any difference in the shape between the sandwich (small spacings) and the reference target (large spacings), the ratio of the corresponding power spectra is computed and shown in Fig. 15(b). Theoretical calculations based on [1] are performed for the two target assemblies, and the ratio of the results can also be seen in the figure. Our implementation does not include the theoretical correction of [3], mentioned on page 6 , since numerical estimates indicate that its influence is marginal for the energy and target composition presented here. The distinct peak at $\simeq 1 \mathrm{GeV}$ in the calculations is clearly absent in the data. It seems that the best description of the data is that there is no effect originating from bringing the foils closer together.

\section{CONCLUSION}

In conclusion, we have observed that as the target thickness increases for the radiation emission from ultrarelativistic electrons, the distorted Coulomb field of the electron which is the result of the first scattering events, leads to a suppressed radiation emission per interaction upon subsequent scattering events. In that case, the radiation intensity becomes proportional to a simple logarithmic function of the thickness, due to the suppression. An animation, showing the time-evolution of the distorted field lines, can be found online [38].

Also, good accordance between data and simulations for the LPM effect in aluminum has been found. On the other hand, the theoretically predicted structure, which should arise when bringing the foils of a structured target closer, cannot be seen in our measurements.

\section{ACKNOWLEDGMENTS}

We are very grateful for the strong support from P. B. Christensen and P. Aggerholm (DPA, Aarhus). We thank S. Fomin and N. Shul'ga for turning our attention to the logarithmic thickness dependence.

\section{APPENDIX A: THE LOGARITHMIC THICKNESS DEPENDENCE}

Blankenbecler's expression $F$ describing the modification to the Bethe-Heitler value, can in the limit $\ell_{\mathrm{f}} \gg \delta t$ and for soft photons be approximated by [[2], Eq. (30)]

$$
\begin{aligned}
& F\left(\ell_{\mathrm{f}} \gg \delta t, T_{\mathrm{B}}, y \ll 1\right) \\
& \simeq \frac{1}{2 T_{\mathrm{B}}} \int_{0}^{1}\left(\frac{3 T_{\mathrm{B}}+1}{1+6 T_{\mathrm{B}} w(1-w)}-1\right) d w,
\end{aligned}
$$

where $T_{\mathrm{B}}$ is a scaled variable of the thickness in units of radiation lengths, $T_{\mathrm{B}}=\frac{\pi}{3 \alpha} \frac{\delta t}{X_{0}}$. Solving the right-hand side (RHS) integration analytically yields

$$
\mathrm{RHS}=\frac{1}{2 T_{\mathrm{B}}}\left(\frac{\operatorname{arccosh}(a)}{\sqrt{1-a^{-2}}}-1\right),
$$



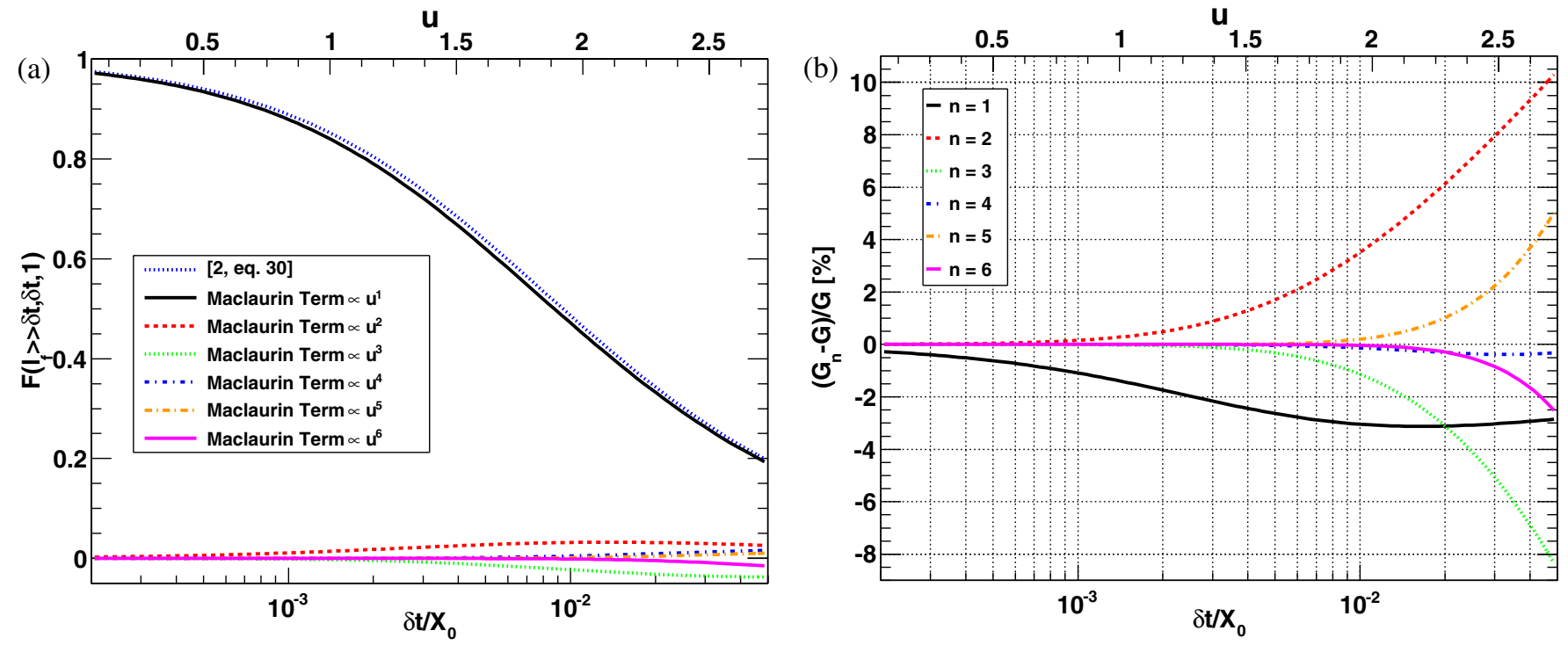

FIG. 16 (color online). Characteristics of the logarithmic expression. (a) Terms of the Maclaurin series. (b) Relative deviation between the truncated Maclaurin series and Eq. (A2).

where $a=3 T_{\mathrm{B}}+1$. In our experiment, the variables have the ranges $T_{\mathrm{B}} \in\left[4.3 \times 10^{-2} ; 7.2\right]$ and $a \in[1.1 ; 23]$. The size of the ranges renders it difficult to find a series expansion in either $a$ or $T_{\mathrm{B}}$ that approximates the full expression above acceptably within the first few terms. Instead, the logarithmic variable $u=\ln \left(2 T_{\mathrm{B}}+1\right)=$ $\ln (2 / 3 a+1 / 3), u \in\left[8.3 \times 10^{-2} ; 2.7\right]$ is introduced. The expression can now be rewritten

$$
F\left(\ell_{\mathrm{f}} \gg \delta t, T_{\mathrm{B}}, y \ll 1\right) \simeq \frac{1}{2 T_{\mathrm{B}}} \underbrace{\left(\frac{\operatorname{arccosh}\left(\frac{3 e^{u}}{2}-\frac{1}{2}\right)}{\sqrt{1-\left(\frac{3 e^{u}}{2}-\frac{1}{2}\right)^{-2}}}-1\right)}_{G(u)} .
$$

If the function $G(u)$ is written in a Maclaurin series, the terms are

$$
\begin{aligned}
G(u) & =\sum_{n=0}^{\infty} \frac{G^{(n)}(0)}{n !} u^{n} \\
& =u+\frac{u^{2}}{20}-\frac{11 u^{3}}{420}+\frac{u^{4}}{240}+\frac{19 u^{5}}{18480}-\frac{3859 u^{6}}{7207200}+\cdots
\end{aligned}
$$

In Fig. 16(a), the contribution from the first six nonvanish- ing terms of the series expansion is shown and compared with Eq. (A2). As can be seen here, substituting $G(u)$ in Eq. (A3) with the first term of the series expansion, leading to $u / 2 T_{\mathrm{B}}=\ln \left(2 T_{\mathrm{B}}+1\right) / 2 T_{\mathrm{B}}$, is a very good approximation to the expression for the factor $F$ in the limit $\ell_{\mathrm{f}} \gg \delta t$. We define $G_{n}(u)$ to be the truncated Maclaurin series neglecting terms of order $u^{n+1}$, thus $G(u) \approx G_{n}(u)+$ $\mathcal{O}\left(u^{n+1}\right)$. In Fig. 16(b), the relative deviation between Eq. (30) of [2] and $G_{n}(u)$ is shown. Within the selected thickness range, $G_{1}(u)=u$ deviates only $3 \%$ from $G(u)$, only surpassed by truncated Maclaurin series with $n \geq 4$. Within the experimental range of thicknesses, the soft power spectrum level with $\ell_{\mathrm{f}} \gg \delta t$ should thus-from a theoretical point of view-be well described by

$$
\begin{aligned}
\frac{X_{0}}{\delta t} \frac{1}{N_{e}} \frac{d N_{\gamma}}{d \ln \hbar \omega} & =\overbrace{\left.\frac{X_{0}}{\delta t} \frac{1}{N_{e}} \frac{d N_{\gamma}}{d \ln \hbar \omega}\right|_{\mathrm{BH}}}^{a} \times F \\
& \simeq a \times \frac{u}{2 T_{\mathrm{B}}}=a \times \frac{\ln (b \times \delta t+1)}{b \times \delta t},
\end{aligned}
$$

where $a$ is close to unity and slightly dependent on $\hbar \omega$, and $b=2 \pi / 3 \alpha X_{0} \simeq 287 / X_{0}$. This expression is identical to Eq. (6) divided by $\delta t / X_{0}$.
[1] R. Blankenbecler and S. D. Drell, Phys. Rev. D 53, 6265 (1996).

[2] R. Blankenbecler, Phys. Rev. D 55, 190 (1997).
[3] R. Blankenbecler, Phys. Rev. D 55, 2441 (1997).

[4] B. G. Zakharov, JETP Lett. 64, 781 (1996).

[5] V. N. Baier and V. M. Katkov, Phys. Rev. D 60, 076001 
(1999).

[6] R. Y. Tsien, Am. J. Phys. 40, 46 (1972).

[7] H. C. Ohanian, Am. J. Phys. 48, 170 (1980).

[8] N. F. Shul'ga and S. P. Fomin, JETP Lett. 27, 117 (1978).

[9] S. P. Fomin and N. F. Shul'ga, Phys. Lett. A 114, 148 (1986).

[10] E. L. Feinberg, Sov. Phys. JETP 23, 132 (1966).

[11] B. M. Bolotovskii and A. V. Serov, Phys. Usp. 40, 1055 (1997).

[12] V.N. Baier and V.M. Katkov, Phys. Rev. D 57, 3146 (1998).

[13] H. Bethe and W. Heitler, Proc. R. Soc. A 146, 83 (1934).

[14] Y.-S. Tsai, Rev. Mod. Phys. 46, 815 (1974).

[15] M. L. Ter-Mikaelian, JETP 25, 296 (1953).

[16] M.L. Ter-Mikaelian, High Energy Electromagnetic Processes in Condensed Matter (John Wiley \& Sons, New York, 1972).

[17] U. I. Uggerhøj, Rev. Mod. Phys. 77, 1131 (2005).

[18] S. Klein, Rev. Mod. Phys. 71, 1501 (1999).

[19] H. D. Hansen et al., Phys. Rev. Lett. 91, 014801 (2003).

[20] H. D. Hansen et al., Phys. Rev. D 69, 032001 (2004).

[21] X.-N. Wang, M. Gyulassy, and M. Plümer, Phys. Rev. D 51, 3436 (1995).

[22] E. Nerush and I. Kostyukov, Phys. Rev. E 75, 057401 (2007).
[23] P. Jaikumar et al., Phys. Rev. D 70, 023004 (2004).

[24] T. Harko and K. S. Cheng, Astrophys. J. 622, 1033 (2005).

[25] A. B. Migdal, Phys. Rev. 103, 1811 (1956).

[26] J. D. Jackson, Classical Electrodynamics (John Wiley \& Sons, New York, 1998), 3rd ed..

[27] F. F. Ternovskii, Sov. Phys. JETP 12, 123 (1961).

[28] U. I. Uggerhøj et al., Phys. Rev. D 72, 112001 (2005).

[29] N.F. Shul'ga and S.P. Fomin, Nucl. Instrum. Methods Phys. Res., Sect. B145, 73 (1998).

[30] P. L. Anthony et al., Phys. Rev. D 56, 1373 (1997).

[31] N. F. Shul'ga and S. P. Fomin, JETP Lett. 63, 873 (1996).

[32] H. D. Thomsen et al., Phys. Lett. B 672, 323 (2009).

[33] N.F. Shul'ga and S. P. Fomin, Sov. Phys. JETP 86, 32 (1998).

[34] B. Z. Kopeliovich, A. Schäfer, and A. V. Tarasov, Phys. Rev. C 59, 1609 (1999).

[35] A. Mangiarotti, S. Ballestrero, P. Sona, and U. Uggerhøj, Nucl. Instrum. Methods Phys. Res., Sect. B 266, 5013 (2008).

[36] V. N. Baier and V.M. Katkov, Phys. Rev. D 59, 056003 (1999).

[37] http://www.phys.au.dk/ ulrik/tsf_animation.gif.

[38] http://www.phys.au.dk/ ulrik/tsf_fieldlines.gif. 